\title{
Multirate Timestepping for the Incompressible Navier-Stokes Equations in Overlapping Grids
}

\author{
Ketan Mittal ${ }^{1, *}$, Som Dutta ${ }^{2}$, Paul Fischer ${ }^{1,3}$
}

\begin{abstract}
We develop a multirate timestepper for semi-implicit solutions of the unsteady incompressible Navier-Stokes equations (INSE) based on a recently-developed multidomain spectral element method (SEM) [1]. For incompressible flows, multirate timestepping (MTS) is particularly challenging because of the tight coupling implied by the incompressibility constraint, which manifests as an elliptic subproblem for the pressure at each timestep. The novelty of our approach stems from the development of a stable overlapping Schwarz method applied directly to the Navier-Stokes equations, rather than to the convective, viscous, and pressure substeps that are at the heart of most INSE solvers. Our MTS approach is based on a predictor-corrector (PC) strategy that preserves the temporal convergence of the underlying semi-implicit timestepper. We present numerical results demonstrating that this approach scales to an arbitrary number of overlapping grids, accurately models complex turbulent flow phenomenon, and improves computational efficiency in comparison to singlerate timestepping-based calculations.
\end{abstract}

Keywords: Multirate, Navier-Stokes, High-order, Nonconforming, Overset

\section{Introduction}

Computational simulations, driven by the improved accessibility of high-performance computing resources, have become ubiquitous for modeling and understanding complex flow phenomena. The accuracy of these computational simulations primarily depends on two key aspects of the method used to solve the partial differential equations (PDE) of interest; (i) the spatial discretization and (ii) the temporal discretization. For spatial discretization, various methods like finite difference (FD), finite element method (FEM), finite volume method (FVM), and the spectral element method (SEM) have become popular for representing the solution of the PDE on a discrete set of nodes/volumes/elements covering the domain $\Omega$ [2, 3, 4]. The spatial accuracy of the discrete solution obtained using these methods depends on the size of the local grid/mesh spacing and the order of accuracy of the spatial discretization. Similarly, the temporal accuracy of the solution depends on the timestep

${ }^{*}$ Corresponding author. Present Address: CASC, Lawrence Livermore National Laboratory, 7000 East Avenue, Livermore, CA 94550

${ }^{1}$ Mechanical Science \& Engineering, University of Illinois at Urbana-Champaign, 1206 W. Green St., Urbana, IL 61801

${ }^{2}$ Mechanical \& Aerospace Engineering, Utah State University, 4130 Old Main Hill, Logan, UT 84332

${ }^{3}$ Computer Science, University of Illinois at Urbana-Champaign, 201 N. Goodwin Ave., Urbana, IL 61801 
size $(\Delta t)$ and the order $(k)$ of the timestepper used for temporal integration. Some popular methods for time-integration include the Runge-Kutta (RKk) method, the Adams-Bashforth $(\mathrm{AB} k)$ method, and the backward differentiation formula (BDFk) method. The focus of this work is on the temporal integration of the incompressible Navier-Stokes equations for modeling fluid dynamics and heat transfer in complex domains.

For the unsteady Navier-Stokes equations (NSE), the nonlinear convective term is typically treaded explicitly [5] and the maximum allowable timestep size for stable time-integration is determined by the Courant-Friedrichs-Lewy (CFL) number [6]. For a problem in $d$ space dimensions, a local CFL at each gridpoint gridpoint $\mathbf{x}_{\mathbf{i}}$ is defined as,

$$
\mathrm{CFL}_{i}:=\left(\sum_{j=1}^{d}\left|\frac{\left(c_{j}\right)_{i}}{\left(\Delta x_{j}\right)_{i}}\right|\right) \Delta t,
$$

where, $\left(c_{j}\right)_{i}$ is the $j$ th component of velocity and $\left(\Delta x_{j}\right)_{i}$ is an approximate grid spacing in the $\mathbf{e}_{j}$ direction, and $\Delta t$ is the timestep size. Using the local CFL, a global CFL for the mesh is defined as

$$
\mathrm{CFL}:=\max _{x_{i}} \mathrm{CFL}_{i} \text {. }
$$

To within a scaling factor, the CFL is a robust and easily evaluated surrogate for $\rho(C) \Delta t$, where $\rho(C)$ is the spectral radius of the (assumed skew-symmetric) convection operator. The CFL limit associated with explicit time-advancement of the convection operator is governed by the scale factor, $G$, such that $\rho(C) \Delta t=G \cdot \mathrm{CFL}$ and the chosen timestepper. For example, for second-order centered differences on a one-dimensional periodic domain with third-order Adams-Bashforth timestepping, we have $G=1$ and $C F L<0.72362$. For Fourier methods, $G=\pi$, and for the SEM, $G \approx 1.2$ for $N>10[4]$.

Most numerical approximations use the same timestep size throughout the domain. These methods are classified as singlerate timestepping (STS) methods. A well-known challenge in STS-based methods, due to the nature of (2), is that even a single point in the domain having a high speed-to-grid-size ratio can have the undesirable effect of limiting the allowable timestep size throughout the domain. This situation occurs, for example, near airfoil trailing edges where flow speeds are high and computational meshes are often dense. Another common case is in the simulation of buoyant plumes [7], as illustrated in Fig. 1. Figure 1(a) shows the structure of a thermally-buoyant plume, Fig. 1(b)-(c) show the monodomain spectral element mesh, and Fig. 1(e) shows the instantaneous velocity magnitude contours. Here, resolution requirements for the turbulence in and near the inlet pipe result in having the finest $(\min \Delta \mathbf{x})$ mesh in the region where flow speed is the highest. Away from the inlet pipe, the turbulence intensity is lower and the meshes are correspondingly coarser. Consequently, we observe that the local CFL is almost two to three orders of magnitude higher for the elements in the plume region as compared to elements in the far-field. The CFL variation throughout the domain is shown on a log-scale in Fig. 11(f). Using the same timestep size for integrating the NSE, thus leads to unnecessary computational cost for elements in the far-field.

The scale disparity in CFL is even more evident when nonconforming overlapping grids are used for this domain (Fig. 1(e)). It is well known that overlapping grids are highly effective in reducing the computational cost of calculations for domains featuring flow structures 


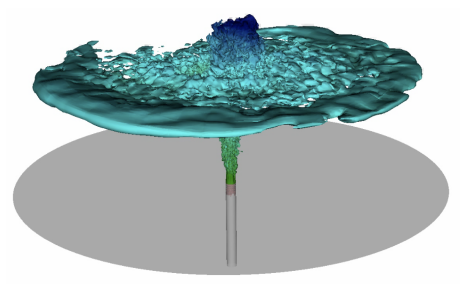

(a) Structure of a thermally-buoyant plume

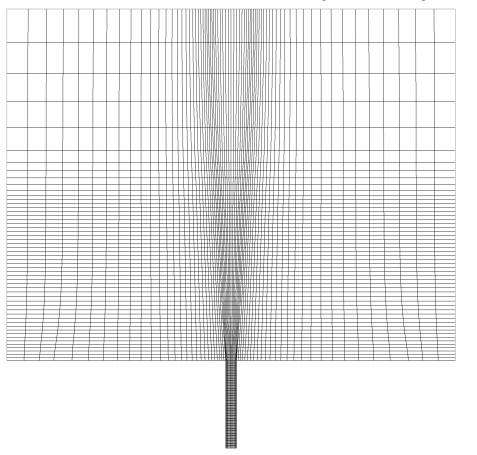

(c) Mondomain grid (slice-view)

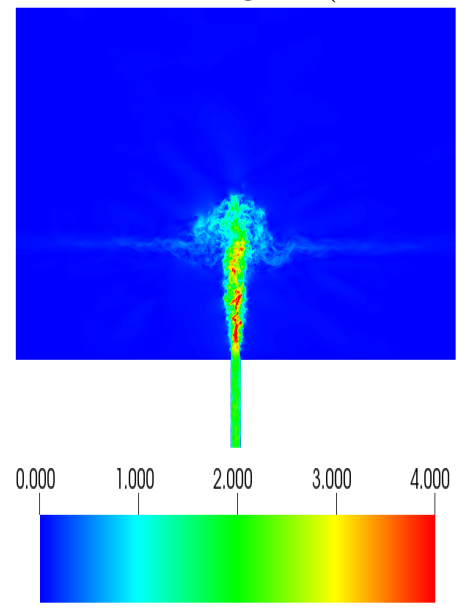

(e) Velocity magnitude

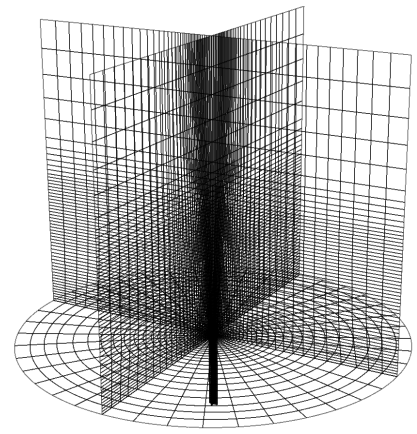

(b) Monodomain grid

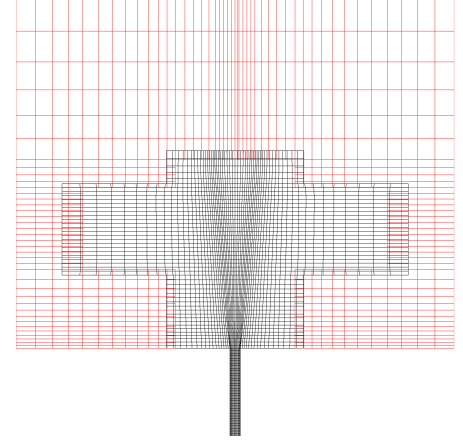

(d) Overlapping grids (slice-view)
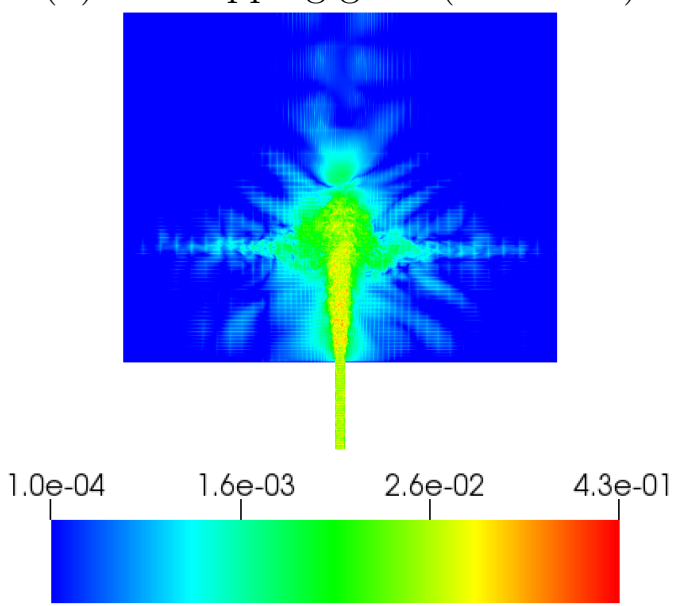

(f) CFL

Figure 1: (a) Structure of a thermally-buoyant plume in a stably stratified environment, (b) monodomain spectral element mesh, slice-view of the (c) monodomain mesh, (d) nonconforming overlapping meshes, (e) velocity magnitude plot, and (f) CFL distribution (log-scale) for a given timestep size. 
with widely varying spatial scales in different regions of the domain. Since overlapping grids relax the constraint of mesh conformity, they reduce the total element count $(10 \%$ in this case) with grids that are constructed according to the physics in the region that they cover. In this example, since the outer grid is much coarser than the inner grid, the outer grid should be able to use orders of magnitude bigger timestep size than the inner grid. Most overlapping grid-based methods, however, use an STS-based approach [1, 8, 9], which results in superfluous computational work for the outer grid.

Multirate timestepping methods were first introduced in the seminal work of Rice in 1960 [10]. Rice developed a Runge-Kutta based timestepping strategy for solving a system of two ordinary differential equations (ODEs) with different integration step sizes. Shortly after, multirate timestepping methods were popularized by Gear [11, 12] and Andrus [13, 14]. In this pioneering work, Gear analyzed the stability and accuracy of Euler method-based strategies for MTS. Similarly, Andrus derived conditions for absolute stability of a highorder Runge-Kutta-based approach for MTS in a system of first-order ODEs. The methods developed by Gear and Andrus were slowest-first- or fastest-first-based, where the ODE with the slower component is solved first followed by the ODE with the faster component, or vice-versa. Other similar works include Gunther's multirate partitioned Runge-Kutta-based (slowest-first) scheme for solving a system of ODE with stiff components [15], Verhoeven's stability analysis of BDF-based (slowest-first) MTS methods for understanding the time behavior of electrical circuits [16], and Godel's fastest-first Adams-Bashforth-based scheme for simulation of electromagnetic wave propagation [17].

While generally useful, a drawback of slowest-first or fastest-first schemes is that they limit the parallelism of the calculation since the ODEs/PDEs are integrated sequentially. In [18, Engstler proposed a method based on Richardson extrapolation to simultaneously solve for the slow and the fast-moving components of a system of ODEs. Similarly, for PDEs, Dawson [19], Constantinescu [20], and Seny [21] have developed parallel MTS methods for solving hyperbolic or parabolic equations with different timestep size for different element groups in a mesh, based on the local CFL number.

Other notable developments in the area of MTS methods include [22, 23, 24, 25, 26, 27]. In [22], Savcenco introduced a novel approach for MTS where all the equations in a system of ODEs are first integrated using a large global timestep size everywhere in the domain, followed by error indicators to determine the equations that require a smaller timestep size. This approach thus avoids unnecessary computation by using a smaller timestep size only for ODEs that require it. Rybak [23] has proposed an MTS method for solving fluid flow in coupled free flow domain and porous media. In [23], the PDE for the free flow domain (INSE) is first temporally integrated using a CFL-dependent smaller timestep size, followed by a larger timestep size to solve the PDE for porous media. While Rybak's and Savcenco's approaches are effective for MTS, they are sequential and lack parallelism, similar to the slowest-first- and fastest-first-based methods. In [24], Emmet has used different timestep sizes for solving fluid motion and relatively stiff chemical mechanism to model compressible reacting flow with complex chemistry. This approach can also be extended to conjugate heat transfer problems where the time scale associated with the energy transfer in fluid and solid medium are very different. Trahan [25] has developed a fastest-first approach for solving the shallow water equations in monodomain conforming grids, Gupta et al. [26] use multirate timestepping for modeling subsurface methane hydrate reservoirs, and Mikida et al. [27] 
solve the compressible Navier-Stokes equations using different timestep sizes in overlapping grids with a fastest-first Adams-Bashforth-based scheme.

A survey of the literature shows multirate timestepping methods have mainly been developed for parabolic and hyperbolic problems [12, 16, 18, 20, 21, 23, 24, 25, 27, 28]. MTS methods are virtually nonexistent for the incompressible Navier-Stokes equations because the solution is sensitive to the pressure, which satisfies an elliptic Poisson problem at every timestep [4]. Since the characteristic propagation speed of pressure perturbations is infinite in incompressible flows, existing approaches for multirate timestepping do not extend to a single conforming mesh. Overlapping grids however, decouple the pressure Poisson solve across the different grids modeling a domain, which allows us to develop a multirate timestepping method. Note that while the MTS method of [23] pertains to INSE, it solves the INSE with a fixed timestep size in the entire domain followed by a different timestep size for the shallow water equations.

In the current work, we develop a parallel multirate timestepping strategy where the INSE are integrated simultaneously in all the overlapping grids. This method circumvents the difficulty of the global divergence-free constraint through a combination of stable high-order predictor-corrector time integrators and mass-flux corrections for time advancement of the unsteady Stokes problems. The nonlinear terms continue to be treated explicitly in time, as in the case of single conforming domain, but are now advanced without the widely disparate values in CFL throughout the global domain. The method scales to an arbitrary number of overlapping grids and supports arbitrarily high (integer) timestep size ratio. Additionally, the approach presented in this paper is agnostic to the spatial discretization (FEM, FVM, SEM, etc.) and can be readily integrated into existing solvers.

The remainder of the paper is organized as follows. Section 2 summarizes the monodomain and overlapping grid-based framework for solving the incompressible Navier-Stokes equations using an STS-based approach [1, 5]. Section 3 builds upon the STS-based method to describe the MTS-based approach for solving the INSE in overlapping grids. In Section 4. we demonstrate that this novel MTS-based approach maintains the temporal accuracy of the underlying $\mathrm{BDF} k / \mathrm{EXT} k$-based timestepper and accurately models complex turbulent flow and heat transfer phenomenon. Here, we also demonstrate that multirate timestepping reduces the computational cost of a calculation in comparison to the STS-based approach. Finally, in Section 5 we discuss some directions for future work.

\section{Preliminaries}

This section provides a description of the singlerate timestepping-based framework for solving the incompressible Navier-Stokes equations in mono- and multi-domain settings, with the latter based on overlapping grids.

\subsection{Governing Equations}

We consider the incompressible Navier-Stokes equations in nondimensional form,

$$
\begin{array}{r}
\frac{\partial \mathbf{u}}{\partial t}+\mathbf{u} \cdot \nabla \mathbf{u}=-\nabla p+\frac{1}{R e} \nabla^{2} \mathbf{u}+\mathbf{f} \\
\nabla \cdot \mathbf{u}=0
\end{array}
$$


where $\mathbf{u}(\mathbf{x}, t)$ and $p(\mathbf{x}, t)$ represent the unknown velocity and pressure that are a function of position ( $\mathbf{x})$ and time $(t)$, and $\mathbf{f}(\mathbf{x}, t)$ is the prescribed forcing. Here, $R e=L U / \nu$ is the Reynolds number based on the characteristic length scale $L$, velocity scale $U$, and kinematic viscosity of the fluid $\nu$. In addition to the INSE, we also consider the energy equation

$$
\frac{\partial T}{\partial t}+\mathbf{u} \cdot \nabla T=\frac{1}{P e} \nabla^{2} T+q_{T}
$$

where $T(\mathbf{x}, t)$ represent the temperature solution and $q_{T}(\mathbf{x}, t)$ is an energy source term. $P e=1 /(R e \cdot P r)$ is the Peclet number, which depends on the Reynolds number and the Prandtl number. The Prandtl number $(\operatorname{Pr}=\nu / \alpha)$ is the ratio of the momentum diffusivity $(\nu)$ and the thermal diffusivity $(\alpha)$. The solution of (3)-(5) also depends on the initial conditions (for time-dependent problems) and boundary conditions.

\subsection{Spatial Discretization}

The multirate timestepping method presented in this work can be readily used with any spatial discretization such as FDM, FEM, and SEM. All the results presented in this paper are based on the SEM [29], which is a high-order weighted residual method that combines the geometric flexibility of finite elements ( $\Omega$ is decomposed into $E$ smaller elements) with the rapid convergence of spectral methods. The basis functions in the SEM are $N$ th-order tensorproduct Lagrange polynomials on the Gauss-Lobatto-Legendre (GLL) quadrature points inside each element. Due to this tensor-product configuration, all operators in SEM can be expressed in a factored matrix-free form, which leads to fast operator-evaluation $\left(O\left(N^{d+1}\right)\right)$ and low operator-storage $\left(\mathrm{O}\left(N^{d}\right)\right)$. The method requires only $C^{0}$ function continuity at element interfaces yet yields exponential convergence of the solution with $N$, resulting in a flexible method with low numerical dispersion. The reader is referred to [4] for a detailed description of the SEM. Note that from a timestepping perspective, the global CFL of each domain is computed by considering the local CFL associated with all the GLL quadrature points inside each element (1). Additionally, throughout this paper, we will focus on the temporal discretization and assume that spatially the grids have been constructed such that they adequately capture the physics of the flow in the region that they cover.

\subsection{Solution of the INSE in a Monodomain Grid}

In our framework, we solve the unsteady INSE in velocity-pressure form using semiimplicit $\mathrm{BDF} k / \mathrm{EXT} k$ timestepping in which the time derivative is approximated by a $k$ thorder backward difference formula (BDFk), the nonlinear terms (and any other forcing) are treated with a $k$ th-order extrapolation $(\mathrm{EXT} k)^{4}$, and the viscous and pressure terms are treated implicitly. This approach leads to a linear unsteady Stokes problem to be solved at each timestep, which is split into independent viscous and pressure (Poisson) updates [5].

Assuming the solution is known at $t^{n-1}$ and that a constant timestep size $\Delta t$ is used for all timesteps, we compute a tentative velocity field at time $t^{n}$ with contributions from the

\footnotetext{
${ }^{4}$ From here on, we will use $k$ to represent the order of accuracy of our temporal discretization, unless otherwise stated.
} 
$\mathrm{BDF} k$ and the explicit terms as

$$
\dot{\mathbf{u}}^{n}=-\sum_{j=1}^{k} \beta_{j} \mathbf{u}^{n-j}+\Delta t \sum_{j=1}^{k} \alpha_{j} \mathbf{f}_{e}^{n-j},
$$

where we use $\mathbf{f}_{e}$ to represent the explicit contributions

$$
\mathbf{f}_{e}^{n-j}=(-\mathbf{u} \cdot \nabla \mathbf{u}+\mathbf{f})^{n-j},
$$

the superscript ()$^{n-j}$ indicates quantities evaluated at earlier timesteps, $t^{n-j}$, and $\beta_{j}$ and $\alpha_{j}$ are the BDF and EXT coefficients, respectively. $\dot{\mathbf{u}}^{n}$ constitutes the nonlinear update but does not account for the divergence-free constraint or viscous effects. The divergencefree constraint (4) is enforced through a pressure correction. A pressure Poisson equation is obtained by taking the divergence of the momentum equation, assuming the solution is divergence-free at time $t^{n}, \nabla \cdot \mathbf{u}^{n}=0$, and using the identity $\nabla^{2} \mathbf{u}^{n}=\nabla\left(\nabla \cdot \mathbf{u}^{n}\right)-\nabla \times \nabla \times \mathbf{u}^{n}$ :

$$
\begin{aligned}
-\nabla^{2} p^{n} & =-\frac{\nabla \cdot \dot{\mathbf{u}}^{n}}{\Delta t}+\frac{1}{R e} \nabla \cdot \sum_{j=1}^{k} \alpha_{j}\left(\nabla \times \boldsymbol{\omega}^{n-j}\right), \\
\Longrightarrow-\nabla^{2} p^{n} & =\nabla \cdot \mathbf{f}_{p},
\end{aligned}
$$

where $\boldsymbol{\omega}^{n}=\nabla \times \mathbf{u}^{n}$, and

$$
\mathbf{f}_{p}=-\frac{\dot{\mathbf{u}}^{n}}{\Delta t}+\frac{1}{R e} \sum_{j=1}^{k} \alpha_{j}\left(\nabla \times \boldsymbol{\omega}^{n-j}\right) .
$$

The advantage of using the curl-curl form for the viscous term to decouple the velocity and pressure solve is that the equation governing the error in divergence $\left(\nabla \cdot \mathbf{u}^{n}\right)$ is an elliptic PDE instead of a parabolic PDE. As a result, this formulation is stable with splitting-induced divergence errors that are only $\mathcal{O}\left(\Delta t^{k}\right)[5,30]$.

Substituting the pressure solution $p^{n}$ in (3), $\mathbf{u}^{n}$ is obtained by solving the Helmholtz equation

$$
\frac{\beta_{0}}{\Delta t} \mathbf{u}^{n}-\frac{1}{R e} \nabla^{2} \mathbf{u}^{n}=-\nabla p^{n}+\frac{\dot{\mathbf{u}}^{n}}{\Delta t} .
$$

Similar to (11), using implicit treatment of the diffusion term and explicit treatment of the advection term for the energy equation, the solution $T^{n}$ for temperature is obtained by solving the Helmholtz equation

$$
\frac{\beta_{0}}{\Delta t} T^{n}-\frac{1}{P e} \nabla^{2} T^{n}=\sum_{j=1}^{k} \frac{\beta_{j}}{\Delta t} \mathbf{u}^{n-j}+\sum_{j=1}^{k} \alpha_{j}\left(-\mathbf{u} \cdot \nabla T+q_{T}\right)^{n-j} .
$$

Spatial discretization of (8)-(12) is based on variational projection operators [4. We impose either essential (Dirichlet) boundary conditions or natural (Neumann) boundary conditions on a surface for velocity (and temperature). As expected, surfaces that have 
Dirichlet conditions for velocity have Neumann conditions for pressure, and vice-versa. Note that we use $\partial \Omega_{D}$ to denote the subset of domain boundary $\partial \Omega$ on which Dirichlet conditions are imposed on velocity and $\partial \Omega_{N}$ for the subset (e.g., outflow) on which pressure is prescribed.

The Navier-Stokes solution time-advancement can be summarized as:

1. Compute the tentative velocity field $\dot{\mathbf{u}}^{n}$ using (6), which accounts for the BDF $k$ and time extrapolated nonlinear terms (EXT $k$ terms).

2. Solve the linear Stokes subproblems (8) and (11) to compute the velocity-pressure solution, $\phi^{n}=\left[\mathbf{u}^{n}, p^{n}\right]^{T}$

$$
\mathbf{S} \phi^{n}=\mathbf{r}^{n},\left.\quad \mathbf{u}^{n}\right|_{\partial \Omega_{D}}=\mathbf{u}_{b}^{n},\left.\quad p^{n}\right|_{\partial \Omega_{N}}=0 .
$$

Here $\mathbf{r}^{n}$, determined using $\mathbf{u}^{n}$, accounts for all inhomogeneities for both pressure and velocity, given on the right-hand sides of (8) and (11), respectively:

$$
\begin{aligned}
& \mathbf{r}^{n}=\left[\mathbf{r}_{v}^{n}, r_{p}^{n}\right]^{T}, \\
& \mathbf{r}_{v}^{n}=-\nabla p^{n}+\frac{\dot{\mathbf{u}}^{n}}{\Delta t}, \quad r_{p}^{n}=-\frac{\nabla \cdot \dot{\mathbf{u}}^{n}}{\Delta t}+\frac{1}{R e} \nabla \cdot \sum_{j=1}^{k} \alpha_{j}\left(\nabla \times \boldsymbol{\omega}^{n-j}\right) .
\end{aligned}
$$

In (13), $\mathbf{u}_{b}^{n}$ is the prescribed velocity on all Dirichlet surfaces $\left(\partial \Omega_{D}\right)$ of the domain, homogeneous Dirichlet conditions are imposed for pressure on outflow surfaces $\left(\partial \Omega_{N}\right)$, and homogeneous Neumann conditions are imposed for velocity on $\partial \Omega_{N}$. It is straightforward to show that the Neumann conditions for pressure on $\partial \Omega_{D}$ can be represented as a function of the Dirichlet condition for velocity $\left(\mathbf{u}_{b}^{n}\right)[5]$.

From an implementation perspective, the solution to the unsteady Stokes problem is obtained in our framework by first solving the pressure Poisson equation (8) using a three-level p-multigrid accelerated by GMRES [31], followed by a diagonally preconditioned conjugate gradient iteration [4] for the Helmholtz solve associated with each component of the velocity (11). Note that for each of these iterative solves, we use a matrix-free approach enabled by the tensor-product structure of the basis functions in the SEM. Further details on the matrix-free operator evaluation with SEM are provided in Section 2.1 of 32 .

In (13), we have omitted the solution to temperature $(12)$ for brevity since it is similar to the Helmholtz solve for velocity. Note that since we always impose Dirichlet conditions for velocity and pressure on $\partial \Omega_{D}$ and $\partial \Omega_{N}$, respectively, we will omit them in the description of timestepping for overlapping grids.

\subsection{Solution of the INSE on Overlapping Grids}

The overlapping Schwarz method for solving a PDE in overlapping domains was introduced by Schwarz in 1870 33. The decomposition for Schwarz's initial model problem is illustrated in Fig. 2 where the domain $\Omega$ is partitioned into two subdomains, a rectangle $\left(\Omega^{1}\right)$ and a circle $\left(\Omega^{2}\right)$, with nonzero overlap such that $\partial \Omega_{I}^{1}:=\partial \Omega^{1} \subset \Omega^{2}$ and $\partial \Omega_{I}^{2}:=\partial \Omega^{2} \subset \Omega^{1}$. We use $\partial \Omega_{I}^{s}$ to denote the "interdomain boundary", namely the segment of the subdomain boundary $\partial \Omega^{s}$ that is interior to another subdomain. The interdomain boundaries $\partial \Omega_{I}^{1}$ and $\partial \Omega_{I}^{2}$ are highlighted in Fig. 2(b). 

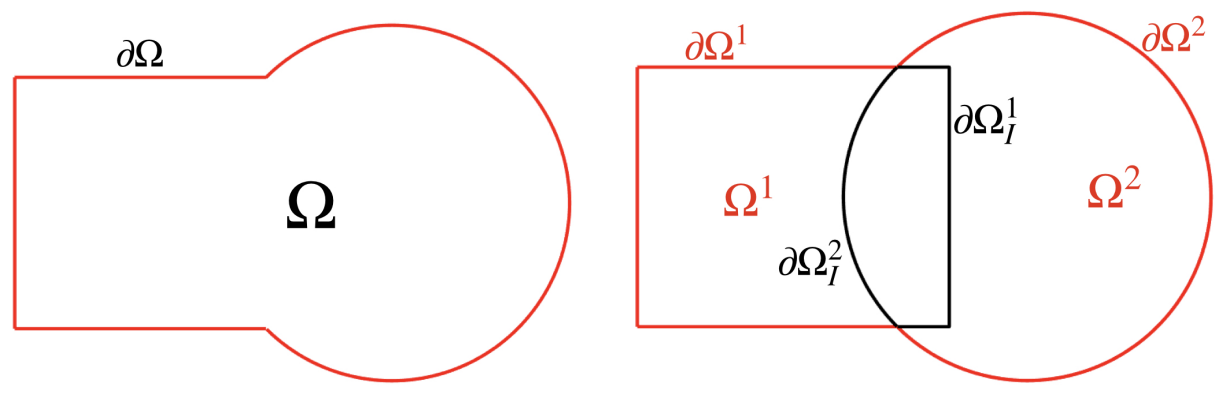

Figure 2: (left to right) (a) Composite domain $\Omega$ (b) modeled by overlapping rectangular $\left(\Omega^{1}\right)$ and circular $\left(\Omega^{2}\right)$ subdomains. $\partial \Omega_{I}^{s}$ denotes the segment of the subdomain boundary $\partial \Omega^{s}$ that is interior to another subdomain $\Omega^{r}$.

There are two key aspects for solving a PDE (INSE) in overlapping grids. First, since overlapping grids introduce interdomain boundaries, a robust mechanism is required to interpolate boundary data for the gridpoints discretizing $\partial \Omega_{I}^{s}$ from the subdomain $\Omega^{r}$ that they overlap. Second, Schwarz iterations are required to ensure that the solution is consistent across the different overlapping grids.

There are two popular approaches for Schwarz iterations. In the alternating Schwarz method, given $S$ overlapping subdomains, the PDE in solved in the first subdomain and that solution is used to update the interdomain boundary data in all other subdomains. This process is repeated sequentially for $s=2 \ldots S$ subdomains. A drawback of the alternating Schwarz method is that it does not scale with the number of subdomains since it requires at-least $S$ steps to obtain the solution of a PDE. In contrast, the simultaneous Schwarz method solves the PDE simultaneously in all subdomains followed by interdomain boundary data exchange. This iterative process is repeated until the solution converges to desired accuracy in the overlap region. Thus, assuming there is a robust mechanism to effect interdomain boundary data exchange, the scalability of the simultaneous Schwarz iterations is not restricted by the number of subdomains. The Schwarz-SEM framework that we describe next is based on the simultaneous Schwarz method, and the reader is referred to [34] for additional details on different OS-based techniques.

For notational purposes, we introduce $\phi^{s, n, q}$ as the solution on the $q$ th Schwarz iteration in subdomain $\Omega^{s}$ at time level $t^{n}$, for $q=0, \ldots, Q$. Thus, assuming that the solution is known up to time $t^{n-1}$ and has been converged using Schwarz iterations at the previous timestep, $\phi^{s, n-1, Q}$ represents the solution at time $t^{n-1}$. With this notation, and assuming a constant timestep size $\Delta t$ (which is equal for all overlapping grids), we define the Schwarz update procedure as follows:

1. Compute the tentative velocity field ú using (6) with the solution from $k$ previous timesteps in each subdomain $\Omega^{s}, s=1 \ldots S$ :

$$
\dot{\mathbf{u}}^{s, n}=-\sum_{j=1}^{k} \beta_{j} \mathbf{u}^{s, n-j, Q}+\Delta t \sum_{j=1}^{k} \alpha_{j} \mathbf{f}_{e}^{s, n-j, Q},
$$

where $\dot{u}^{s, n}$ has contributions from the BDF $k$ and EXT $k$ terms. We note that we do 
not use the superscript ( $)^{q}$ in $\mathbf{u}^{s, n}$ because it depends only on the solution at previous timesteps and does not change at each Schwarz iteration.

2. Use $Q$ simultaneous Schwarz iterations to solve the linear Stokes subproblems (8) and (11) to yield the velocity-pressure pair, $\phi^{s, n, q}=\left[\mathbf{u}^{s, n, q}, p^{s, n, q}\right]^{T}$.

- Prior to each Schwarz iteration, interpolate the interdomain boundary data (global process). Since the solution is known up to $t^{n-1}$, the initial iterate $(q=0$, the predictor step) uses interdomain boundary data based on $m$ th-order extrapolation in time. The $Q$ subsequent Schwarz iterations (the corrector steps) directly interpolate the interdomain boundary data from the most recent iteration:

$$
\begin{aligned}
& q=0:\left.\quad \hat{\mathbf{u}}^{s, n, 0}\right|_{\partial \Omega_{I}^{s}}=\sum_{j=1}^{m} \gamma_{j} \mathcal{I}\left(\mathbf{u}^{r, n-j, Q}\right), \\
& q=1 \ldots Q:\left.\hat{\mathbf{u}}^{s, n, q}\right|_{\partial \Omega_{I}^{s}}=\mathcal{I}\left(\mathbf{u}^{r, n, q-1}\right),
\end{aligned}
$$

where $m$ is the order of extrapolation for the interdomain boundary data at $q=0$ iteration, $\gamma_{j}$ are the corresponding extrapolation weights that are computed using the routines described in [35], and $\mathcal{I}$ is the interpolation operator described in Section 2.4.1. Note that each Schwarz iteration in singlerate timestepping effectively requires 1 interpolation, even though the predictor step $(q=0)$ depends on $m$ most recent solutions (e.g., $\left.\mathbf{u}^{r, n-1, Q}, \mathbf{u}^{r, n-2, Q}, \mathbf{u}^{r, n-3, Q}\right)$. This is because all solutions except the most recent one (e.g., $\mathbf{u}^{r, n-2, Q}$ and $\mathbf{u}^{r, n-3, Q}$ ) are already available since they must have been interpolated at the predictor step of the previous timestep.

- Solve the unsteady Stokes problem (locally) in each subdomain:

$$
q=0 \ldots Q: \mathbf{S} \phi^{s, n, q}=\mathbf{r}^{s, n, q},\left.\quad \mathbf{u}^{s, n, q}\right|_{\partial \Omega_{I}^{s}}=\left.\hat{\mathbf{u}}^{s, n, q}\right|_{\partial \Omega_{I}^{s}}+\left.\tilde{\mathbf{u}}\right|_{\partial \Omega_{I}^{s}},
$$

where $\left.\tilde{\mathbf{u}}\right|_{\partial \Omega_{I}^{s}}$ is a flux-based correction (Section 2.4.2) required to satisfy the divergence-free constraint, and

$$
\begin{aligned}
& \mathbf{r}^{s, n, q}=\left[\mathbf{r}_{v}^{s, n, q}, r_{p}^{s, n, q}\right]^{T}, \\
& \mathbf{r}_{v}^{s, n, q}=-\nabla p^{s, n, q}+\frac{\hat{\mathbf{u}}^{s, n}}{\Delta t}, \\
& r_{p}^{s, n, q}=-\frac{\nabla \cdot \dot{\mathbf{u}}^{s, n}}{\Delta t}+\frac{1}{R e} \nabla \cdot \sum_{j=1}^{k} \alpha_{j}\left(\nabla \times \boldsymbol{\omega}^{s, n-j, Q}\right) .
\end{aligned}
$$

In (18), we solve the full unsteady Stokes problem for velocity and pressure in each subdomain, $\Omega^{s}$. This unsteady Stokes solve is similar to the monodomain case (13), with the addition of a Dirichlet condition on the interdomain boundaries $\left(\partial \Omega_{I}^{s}\right)$ that is obtained by interpolating the solution from the overlapping subdomain (prior to each Schwarz iteration) and imposing a flux-based correction $\left(\left.\tilde{\mathbf{u}}\right|_{\partial \Omega_{I}^{s}}\right)$.

Note that besides the interpolation of the interdomain boundary data, which is a global process and requires communication between overlapping subdomains, the unsteady Stokes 
solve is independent for each subdomain and utilizes the same matrix-free framework that is used for a monodomain grid (Section 2.3).

The temporal accuracy of this singlerate timestepping scheme (18) is $O\left(\Delta t^{\min (m, k)}\right)$. We typically set $m=k$, unless otherwise stated, and Peet and Fischer [36] have shown that $Q=0$ is sufficient for $m=1$ and $Q=1-3$ is sufficient for $m>1$ from a stability point of view. In [1, we further demonstrated that $Q=0$ is sufficient from an accuracy point of view for basic statistics (e.g., mean or rms) of turbulent flows. This predictor-corrector approach has been used in the Schwarz-SEM framework [37, 38, 39] to demonstrate that it maintains the spatial and temporal convergence of the underlying monodomain SEM framework, and is effective for solving highly turbulent flow phenomenon in complex domains using an arbitrary number of overlapping grids.

\subsubsection{Interpolation}

Since overlapping grids rely on interpolation for interdomain boundary data, the interpolation operator $(\mathcal{I})$ is of central significance for overlapping Schwarz based methods. In our framework, $\mathcal{I}$ is effected (in parallel) via findpts, a scalable high-order interpolation utility that is part of gslib [40], an open-source communication library that readily links with Fortran, $\mathrm{C}$, and $\mathrm{C}++$ codes.

findpts provides two key functionalities. First, for a given set of interdomain boundary points that are tagged with the associated subdomain number $\mathbf{x}^{*}=\left(\mathbf{x}_{1}^{*}, \mathbf{x}_{2}^{*} \ldots \mathbf{x}_{b}^{*}\right)^{s}$, findpts determines the computational coordinates of each point. These computational coordinates $(\mathbf{q}=r, e, \boldsymbol{\xi}, p)$ for each point specify the subdomain number $r$ that it overlaps, the element number $\left(e \in \Omega^{r}\right)$ in which the point was found, and the corresponding reference-space coordinates $(\boldsymbol{\xi}=(\xi, \eta, \zeta)))$ inside that element. Since a mesh could be partitioned on to many MPI ranks, findpts also specifies the MPI rank $p$ on which the donor element is located. For cases where $S=2$, the donor element search is straightforward [41] because findpts is only concerned with the elements that are not located in the same subdomain as the sought-point $\left(e \in \Omega^{r}, r \neq s\right)$. In cases where $S>2$, an interdomain boundary point can overlap multiple subdomains. In these cases, the donor element is chosen from the subdomain that minimizes the error due to simultaneous Schwarz iterations [1]. If the nodal positions of all the overlapping grids are fixed in time, the computational coordinate search needs to be done only at the beginning of the calculation. Otherwise, the computational coordinate search is done at the beginning of each time-step.

The second key functionality of findpts is that for a given set of computational coordinates, it can interpolate any scalar function defined on the spectral element mesh. All the parallel communication in findpts is handled by gslib's generalized and scalable all-to-all utility, gs_crystal, which is based on the crystal router algorithm of [42. Using gslib, findpts has demonstrated excellent scaling in parallel for finding computational coordinates of a given set of points and interpolating solution in a mondomain mesh [43] and in overlapping meshes [1].

\subsubsection{Mass-Balance}

While the gslib-based interpolation is exponentially convergent, there can still be mass balance errors arising from the interpolation of the velocities on the interdomain boundaries. Since the pressure solve is sensitive to these inconsistencies, we must ensure that for each 
subdomain $\Omega^{s}$, the following compatibility condition for INSE is satisfied at all times:

$$
\int_{\partial \Omega^{s}} \mathbf{u} \cdot \hat{\mathbf{n}}=0
$$

where $\hat{\mathbf{n}}$ represents the outward pointing unit normal vector on $\partial \Omega^{s}$. In the case of overlapping grids, when a subdomain does not have any natural boundary conditions $\left(\partial \Omega_{N} \cap \partial \Omega^{s}=\right.$ 0 ), there is a potential to fail to satisfy $(20)$ because the interpolated fluxes on $\partial \Omega^{s}$ may not integrate to zero.

Let $\hat{\mathbf{u}}$ denote the tentative velocity field defined on $\partial \Omega^{s}$ through prescribed Dirichlet data on $\partial \Omega_{D}^{s}:=\partial \Omega^{s} \cap \partial \Omega_{D}$ and interpolation on $\partial \Omega_{I}^{s}$. Assume a correction of the form

$$
\left.\mathbf{u}\right|_{\partial \Omega_{I}^{s}}=\left.\hat{\mathbf{u}}\right|_{\partial \Omega_{I}^{s}}+\left.\tilde{\mathbf{u}}\right|_{\partial \Omega_{I}^{s}}
$$

where $\mathbf{u}$ is the flux-corrected boundary data on $\partial \Omega^{s}$ and $\tilde{\mathbf{u}}$ is the correction required to satisfy (20). In [1], we demonstrated that the choice

$$
\left.\tilde{\mathbf{u}}\right|_{\partial \Omega_{I}^{s}}=\left.\delta \hat{\mathbf{n}}\right|_{\partial \Omega_{I}^{s}}
$$

is the $L^{2}$ minimizer of possible trace-space corrections, $(\mathbf{u}-\hat{\mathbf{u}})$, that allows 20$)$ to be satisfied, provided that

$$
\delta=-\frac{\int_{\partial \Omega^{s}} \hat{\mathbf{u}} \cdot \hat{\mathbf{n}} d A}{\int_{\partial \Omega_{I}^{s}} \hat{\mathbf{n}} \cdot \hat{\mathbf{n}} d A}=-\frac{\int_{\partial \Omega_{D}^{s}} \hat{\mathbf{u}} \cdot \hat{\mathbf{n}} d A+\int_{\partial \Omega_{I}^{s}} \hat{\mathbf{u}} \cdot \hat{\mathbf{n}} d A}{\int_{\partial \Omega_{I}^{s}} \hat{\mathbf{n}} \cdot \hat{\mathbf{n}} d A}
$$

Here, the first term in the numerator $\left(\int_{\partial \Omega_{D}^{s}} \hat{\mathbf{u}} \cdot \hat{\mathbf{n}} d A\right)$ is known by definition since it is the prescribed boundary data on $\partial \Omega_{D}^{s}$. The second term, $\int_{\partial \Omega_{I}^{s}} \hat{\mathbf{u}} \cdot \hat{\mathbf{n}} d A$, is based on the interpolated data (16)-17), obtained prior to each Schwarz iteration. Using this flux-correction approach, we modify the interpolated boundary data for each unsteady Stokes solve (18). Note that unlike interdomain interpolation, which is a global process and requires communication between all overlapping subdomains, the flux-based correction is local to each subdomain since it only depends on $\partial \Omega^{s}$.

\section{Methodology for Multirate Timestepping}

In this section, we introduce the parallel multirate timestepping scheme for solving INSE in overlapping subdomains. For simplicity, we first describe our method in the context of two overlapping subdomains with a fixed timestep ratio of 2. Next, we describe our method for an arbitrary timestep ratio, since this is an obvious next step and a scenario that we encounter in most of our applications (e.g., thermally-buoyant plume in Section 1 that is modeled with overlapping domains with a timestep ratio of 100). Finally, we generalize the

MTS scheme to an arbitrary number of overlapping subdomains with an arbitrary timestep ratio.

In our method, we consider only integer timestep ratios,

$$
\eta:=\frac{\Delta t_{c}}{\Delta t_{f}} \in \mathbb{Z}^{+}
$$


where $\Delta t_{c}$ corresponds to the subdomain $\left(\Omega^{c}\right)$ with slower time-scales and $\Delta t_{f}$ corresponds to the subdomain $\left(\Omega^{f}\right)$ with faster time-scales. Figure 3 shows a schematic of the discrete time-levels for the STS scheme and the MTS scheme with $\eta=2$. Here, the black circles (-) indicate the timestep levels for both $\Omega^{f}$ and $\Omega^{c}$ and the blue circles (॰) indicate the sub-timestep levels for $\Omega^{f}$.

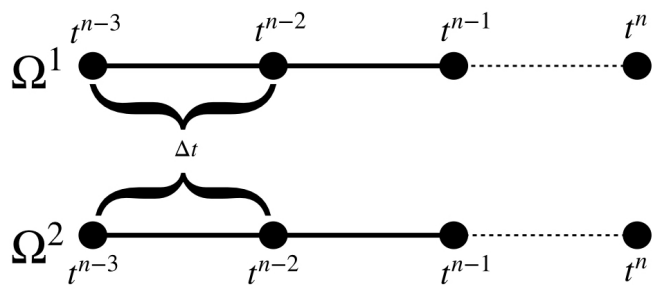

(a) Singlerate timestepping.

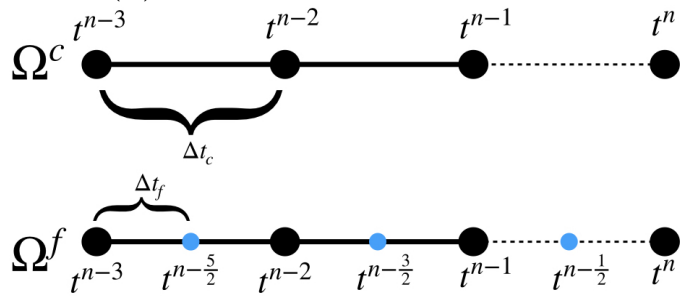

(b) Multirate timestepping with timestep ratio $\eta=2$.

Figure 3: Schematic showing discrete time-levels for singlerate and multirate timestepping $(\eta=2)$.

\subsection{Multirate Timestepping for $\eta=2$}

In a slowest- or fastest-first method, assuming the solution is known up to $t^{n-1}$, the PDE of interest is temporally integrated in either of the domains (e.g., say $\Omega^{f}$ ) to obtain the solution at time $t^{n}$, which is then used to obtain interdomain boundary data for advancing the solution in the other domain (e.g., $\Omega^{c}$ ). For a parallel multirate scheme, however, we wish to simultaneously advance the solution in $\Omega^{f}$ and $\Omega^{c}$. As a result, the interdomain boundary data is exchanged prior to starting the solution process such that the unsteady Stokes solve in each subdomain ( $\eta$ sub-timesteps in $\Omega^{f}$ and 1 timestep in $\Omega^{c}$ ) can be completed independently, similar to the singlerate timestepping (Section 2.4). The synchronization time-levels at which the interdomain boundary data is exchanged are indicated by (0) in Fig. 3.

Similar to the singlerate timestepping scheme (e.g., see (18p), high-order temporal accuracy is achieved in the multirate setting by extrapolating the interdomain boundary data obtained from the solution at previous (sub-) timesteps. For $\eta=2$, the interdomain boundary data dependency for the predictor step is depicted in Fig. 4. For the solutions $\phi^{f, n-\frac{1}{2}, 0}$ and $\phi^{f, n, 0}$ the boundary data is interpolated from the known solutions in $\Omega^{c}: \phi^{c, n-1, Q}, \phi^{c, n-2, Q}$, and $\phi^{c, n-3, Q}$. Simultaneously, the interdomain boundary data for the solution $\phi^{c, n, 0}$ is interpolated from the known solutions in $\Omega^{f}: \phi^{f, n-1, Q}, \phi^{f, n-\frac{3}{2}, Q}$, and $\phi^{f, n-2, Q}$. This interdomain boundary data exchange occurs at synchronization time-level $t^{n-1}$, prior to starting the solution process for times $t^{n-\frac{1}{2}}$ and $t^{n}$. 


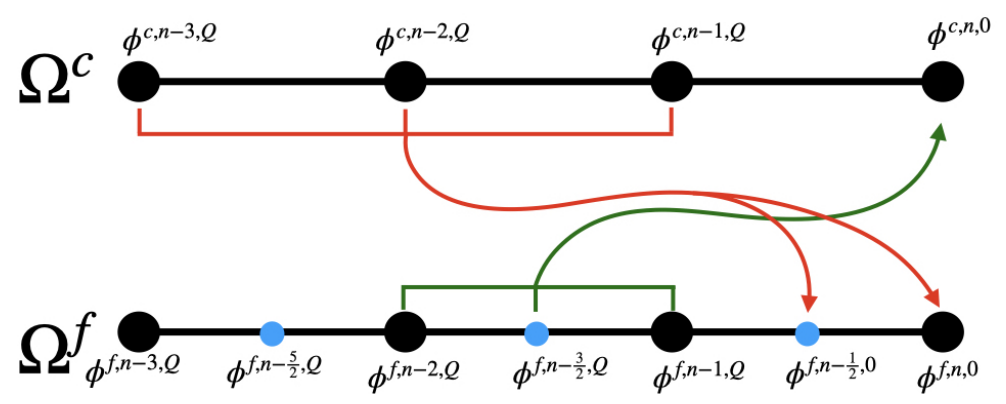

Figure 4: Schematic showing the dependence of the interdomain boundary data for the predictor step.

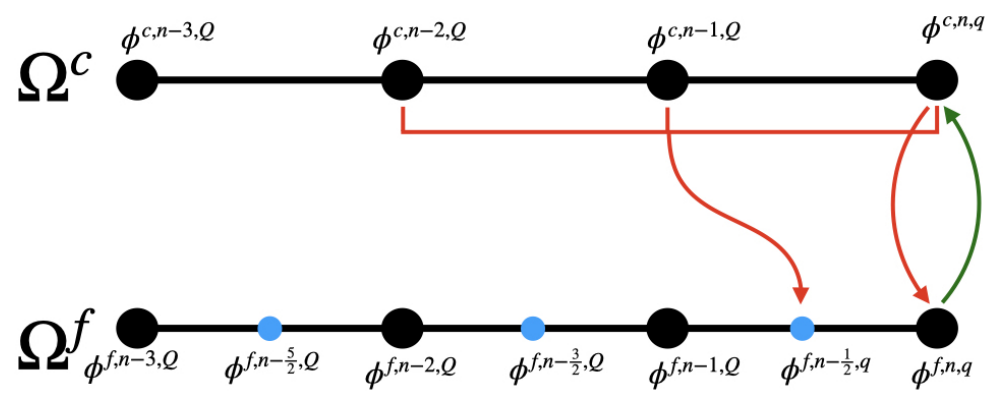

Figure 5: Schematic showing the dependence of the interdomain boundary data for corrector steps.

Once the solution $\phi^{f, n, 0}$ and $\phi^{c, n, 0}$ have been determined, $Q$ correction iterations are needed to stabilize the solution if high-order extrapolation is used for interdomain boundary data during the predictor step. The interdomain boundary data dependency for the corrector steps is depicted in Fig. 5. In $\Omega^{f}$, the interdomain boundary data for $\phi^{f, n-\frac{1}{2}, q}$ comes from the most recent iteration in $\Omega^{c}\left(\phi^{c, n, q-1}\right)$ and the converged solution at previous timesteps, $\boldsymbol{\phi}^{c, n-1, Q}$ and $\boldsymbol{\phi}^{c, n-2, Q}$. For the solution at time $t^{n}\left(\phi^{f, n, q}\right.$ and $\left.\phi^{c, n, q}\right)$, the interdomain boundary data only depends on the solution from the most recent iteration $\left(\phi^{c, n, q-1}\right.$ and $\phi^{f, n, q-1}$ ).

Using this approach for obtaining interdomain boundary data, we now summarize the multirate timestepping scheme for $\eta=2$. Recall our notation for singlerate timestepping, $\phi^{s, n, q}$ denotes the solution $\phi$ in $\Omega^{s}$ at the $q$ th Schwarz iteration at time $t^{n}$.

1. For the predictor step $(q=0)$, assuming that the solution is known up to time $t^{n-1}$, interpolate the interdomain boundary data (global process) followed by locally computing the tentative velocity field $\mathbf{u}(6)$ and solving the linear Stokes problem in each subdomain:

- Interpolate the interdomain boundary data required for each sub-timestep of $\Omega^{f}$ and the 
only timestep of $\Omega^{c}$ :

$$
\begin{gathered}
\left.\hat{\mathbf{u}}^{f, n-\frac{1}{2}, 0}\right|_{\partial \Omega_{I}^{f}}=\mathcal{I}\left(\sum_{j=1}^{m} \gamma_{1 j} \mathbf{u}^{c, n-j, Q}\right), \\
\left.\hat{\mathbf{u}}^{f, n, 0}\right|_{\partial \Omega_{I}^{f}}=\mathcal{I}\left(\sum_{j=1}^{m} \gamma_{2 j} \mathbf{u}^{c, n-j, Q}\right), \\
\left.\hat{\mathbf{u}}^{c, n, 0}\right|_{\partial \Omega_{I}^{c}}=\mathcal{I}\left(\sum_{j=1}^{m} \gamma_{1 j} \mathbf{u}^{f, n-\frac{j+1}{2}, Q}\right) .
\end{gathered}
$$

Here, equations (25) and (26) refer to the interdomain boundary data required for the first and second sub-timestep, respectively, of $\Omega^{f}$, and (27) refers to the interdomain boundary data required for the only timestep of $\Omega^{c}$. In 25$)-(27), \gamma_{i j}$ refers to the coefficients used to extrapolate the interdomain boundary data at the $i$ th sub-timestep, which are computed based on the time-levels (e.g., $t^{n-1}, t^{n-\frac{3}{2}}$, etc.) and the order of extrapolation $m$ such that the desired temporal accuracy is maintained, using the routines described in [35].

- Unsteady Stokes solve for the first sub-timestep in $\Omega^{f}$ :

$$
\begin{aligned}
& \dot{\mathbf{u}}^{f, n-\frac{1}{2}}=-\sum_{j=1}^{k} \beta_{j} \mathbf{u}^{f, n-\frac{j+1}{2}, Q}+\Delta t_{f} \sum_{j=1}^{k} \alpha_{j} \mathbf{f}_{e}^{f, n-\frac{j+1}{2}, Q}, \\
& \mathbf{S} \phi^{f, n-\frac{1}{2}, 0}=\mathbf{r}^{f, n-\frac{1}{2}, 0},\left.\quad \mathbf{u}^{f, n-\frac{1}{2}, 0}\right|_{\partial \Omega_{I}^{f}}=\left.\hat{\mathbf{u}}^{f, n-\frac{1}{2}, 0}\right|_{\partial \Omega_{I}^{f}}+\left.\delta \hat{\mathbf{n}}\right|_{\partial \Omega_{I}^{f}} .
\end{aligned}
$$

- Unsteady Stokes solve for the second sub-timestep in $\Omega^{f}$ :

$$
\begin{aligned}
& \dot{\mathbf{u}}^{f, n}=-\sum_{j=1}^{k} \beta_{j} \mathbf{u}^{f, n-\frac{j}{2}, Q}+\Delta t_{f} \sum_{j=1}^{k} \alpha_{j} \mathbf{f}_{e}^{f, n-\frac{j}{2}, Q}, \\
& \mathbf{S} \phi^{f, n, 0}=\mathbf{r}^{f, n, 0},\left.\quad \mathbf{u}^{f, n, 0}\right|_{\partial \Omega_{I}^{f}}=\left.\hat{\mathbf{u}}^{f, n, 0}\right|_{\partial \Omega_{I}^{f}}+\left.\delta \hat{\mathbf{n}}\right|_{\partial \Omega_{I}^{f}} .
\end{aligned}
$$

- Unsteady Stokes solve for the only timestep in $\Omega^{c}$ :

$$
\begin{aligned}
& \dot{\mathbf{u}}^{c, n}=-\sum_{j=1}^{k} \beta_{j} \mathbf{u}^{c, n-j, Q}+\Delta t_{c} \sum_{j=1}^{k} \alpha_{j} \mathbf{f}_{e}^{c, n-j, Q}, \\
& \mathbf{S} \boldsymbol{\phi}^{c, n, 0}=\mathbf{r}^{c, n, 0},\left.\quad \mathbf{u}^{c, n, 0}\right|_{\partial \Omega_{I}^{c}}=\left.\hat{\mathbf{u}}^{c, n, 0}\right|_{\partial \Omega_{I}^{c}}+\left.\delta \hat{\mathbf{n}}\right|_{\partial \Omega_{I}^{c}} .
\end{aligned}
$$

In (28), we first compute the tentative velocity field, similar to (15) for singlerate timestepping, and then solve for $\phi^{f, n-\frac{1}{2}, 0}$ using the flux-corrected interdomain boundary data (Section 2.4.2). Note that computing $\delta$ for flux-correction through (23) requires the interpolated interdomain boundary data (25)-(27) and the prescribed Dirichlet data on $\partial \Omega_{D}^{s}$ (which is known by definition). Additionally, $\mathbf{r}^{f, n-\frac{1}{2}, q}$ is unchanged from $(19)$ for singlerate timestepping scheme.

Once $\phi^{f, n-\frac{1}{2}, 0}$ is determined, $\phi^{f, n, 0}$ is computed using $(29)$, which completes the predictor step for advancing the solution of INSE in $\Omega^{f}$. Parallel to $(28)$ and $(29),(30)$ is used for the solution at time $t^{n}$ in $\Omega^{c}$. 
2. Once the predictor step, $q=0$, is complete, $q=1 \ldots Q$ corrector iterations are done to improve the accuracy of the solution and stabilize the method:

- Interpolate the interdomain boundary data:

$$
\begin{aligned}
\left.\hat{\mathbf{u}}^{f, n-\frac{1}{2}, q}\right|_{\partial \Omega_{I}^{f}} & =\mathcal{I}\left(\zeta_{11} \mathbf{u}^{c, n, q-1}+\zeta_{12} \mathbf{u}^{c, n-1, Q}+\zeta_{13} \mathbf{u}^{c, n-2, Q}\right), \\
\left.\hat{\mathbf{u}}^{f, n, q}\right|_{\partial \Omega_{I}^{f}} & =\mathcal{I}\left(\mathbf{u}^{c, n, q-1}\right), \\
\left.\hat{\mathbf{u}}^{c, n, q}\right|_{\partial \Omega_{I}^{c}} & =\mathcal{I}\left(\mathbf{u}^{f, n, q-1}\right) .
\end{aligned}
$$

In (31), the coefficients for the temporal interpolation represented by $\zeta_{1 j}$ are computed assuming linear interpolation when $m=1$ or 2 , and quadratic interpolation when $m=3$. This approach ensures that the desired temporal accuracy $\mathcal{O}\left(\Delta t^{m}\right)$ is maintained. In (32) and (33), the interdomain boundary data is directly spatially interpolated without the need for any temporal interpolation.

- Unsteady Stokes solve for the first sub-timestep in $\Omega^{f}$ :

$$
\begin{aligned}
& \dot{\mathbf{u}}^{f, n-\frac{1}{2}}=-\sum_{j=1}^{k} \beta_{j} \mathbf{u}^{f, n-\frac{j+1}{2}, Q}+\Delta t_{f} \sum_{j=1}^{k} \alpha_{j} \mathbf{f}_{e}^{f, n-\frac{j+1}{2}, Q}, \\
& \mathbf{S} \phi^{f, n-\frac{1}{2}, q}=\mathbf{r}^{f, n-\frac{1}{2}, q},\left.\quad \mathbf{u}^{f, n-\frac{1}{2}, q}\right|_{\partial \Omega_{I}^{f}}=\left.\hat{\mathbf{u}}^{f, n-\frac{1}{2}, q}\right|_{\partial \Omega_{I}^{f}}+\left.\delta \hat{\mathbf{n}}\right|_{\partial \Omega_{I}^{f}} .
\end{aligned}
$$

- Unsteady Stokes solve for the second sub-timestep in $\Omega^{f}$ :

$$
\begin{aligned}
& \dot{\mathbf{u}}^{f, n}=-\sum_{j=1}^{k} \beta_{j} \mathbf{u}^{f, n-\frac{j}{2}, Q}+\Delta t_{f} \sum_{j=1}^{k} \alpha_{j} \mathbf{f}_{e}^{f, n-\frac{j}{2}, Q}, \\
& \mathbf{S} \phi^{f, n, q}=\mathbf{r}^{f, n, q},\left.\quad \mathbf{u}^{f, n, q}\right|_{\partial \Omega_{I}^{f}}=\left.\hat{\mathbf{u}}^{f, n, q}\right|_{\partial \Omega_{I}^{f}}+\left.\delta \hat{\mathbf{n}}\right|_{\partial \Omega_{I}^{f}}
\end{aligned}
$$

- Unsteady Stokes solve for the only timestep in $\Omega^{c}$ :

$$
\mathbf{S} \phi^{c, n, q}=\mathbf{r}^{c, n, q},\left.\quad \mathbf{u}^{c, n, q}\right|_{\partial \Omega_{I}^{c}}=\left.\hat{\mathbf{u}}^{c, n, q}\right|_{\partial \Omega_{I}^{c}}+\left.\delta \hat{\mathbf{n}}\right|_{\partial \Omega_{I}^{c}}
$$

In (34), we compute $\phi^{f, n-\frac{1}{2}, q}$ using the flux-corrected interdomain boundary data, followed by (35) to determine $\phi^{f, n, q}$ in $\Omega^{f}$. Similarly, $\phi^{c, n, q}$ is (concurrently) computed using (36).

We note that in the singlerate timestepping scheme, the tentative velocity field (ú) was computed only once for the $Q$ corrector iterations (15). In contrast, we recompute the tentative velocity field in $(34)-(35)$ at each corrector iteration for $\Omega^{f}$, because the solution process spans $\eta$ multiple sub-timesteps. Saving ú for each of the $\eta$ sub-timesteps is not a scalable approach (e.g., $\eta=100$ will require us to save tentative velocity field for 100 subtimesteps). Using (31)-(36), $Q$ simultaneous Schwarz iterations determine the solution in $\Omega^{f}$ and $\Omega^{c}$ at time $t^{n}$. 


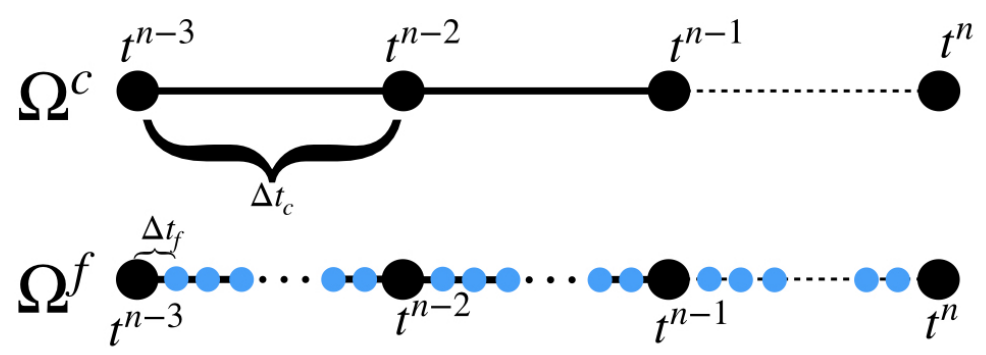

Figure 6: Schematic showing discrete time-levels for the multirate timestepping with an arbitrary timestep ratio.

\subsection{Multirate Timestepping for Arbitrary $\eta$}

From the preceding discussion, we can anticipate that the generalization of this multirate scheme will require $\eta$ sub-timesteps in $\Omega^{f}$ and only one timestep in $\Omega^{c}$. Figure 6 shows a schematic with time-levels for an arbitrary timestep ratio. Similar to (25)-(36), the timestepping strategy for arbitrary (integer) $\eta$ is,

1. Compute the tentative velocity field and solve the linear Stokes problem in each subdomain for the predictor step $(q=0)$.

- Interpolate the interdomain boundary data for $i=1 \ldots \eta$ sub-timesteps of $\Omega^{f}$ and 1 timestep of $\Omega^{c}$ :

$$
\begin{aligned}
\left.\hat{\mathbf{u}}^{f, n-1+\frac{i}{\eta}, 0}\right|_{\partial \Omega_{I}^{f}} & =\mathcal{I}\left(\sum_{j=1}^{m} \gamma_{i j} \mathbf{u}^{c, n-j, Q}\right), \quad i=1 \ldots \eta \\
\left.\hat{\mathbf{u}}^{c, n, 0}\right|_{\partial \Omega_{I}^{c}} & =\mathcal{I}\left(\sum_{j=1}^{m} \gamma_{1 j} \mathbf{u}^{f, n-1-\frac{j-1}{\eta}, Q}\right) .
\end{aligned}
$$

- Unsteady Stokes solve for the $i=1 \ldots \eta$ sub-timesteps of $\Omega^{f}$ :

$$
\begin{aligned}
\mathbf{\mathbf { u }}^{f, n-1+\frac{i}{\eta}} & =-\sum_{j=1}^{k} \beta_{j} \mathbf{u}^{f, n-1-\frac{j-i}{\eta}, Q}+\Delta t_{f} \sum_{j=1}^{k} \alpha_{j} \mathbf{f}_{e}^{f, n-1-\frac{j-i}{\eta}, Q}, \\
\mathbf{S} \phi^{f, n-1+\frac{i}{\eta}, 0} & =\mathbf{r}^{f, n-1+\frac{i}{\eta}, 0},\left.\quad \mathbf{u}^{f, n-1+\frac{i}{\eta}, 0}\right|_{\partial \Omega_{I}^{f}}=\left.\hat{\mathbf{u}}^{f, n-1+\frac{i}{\eta}, 0}\right|_{\partial \Omega_{I}^{f}}+\left.\delta \hat{\mathbf{n}}\right|_{\partial \Omega_{I}^{f} .}
\end{aligned}
$$

- Unsteady Stokes solve for the only timestep of $\Omega^{c}$ :

$$
\begin{aligned}
& \hat{\mathbf{u}}^{c, n}=-\sum_{j=1}^{k} \beta_{j} \mathbf{u}^{c, n-j, Q}+\Delta t_{c} \sum_{j=1}^{k} \alpha_{j} \mathbf{f}_{e}^{c, n-j, Q}, \\
& \mathbf{S} \phi^{c, n, 0}=\mathbf{r}^{c, n, 0},\left.\quad \mathbf{u}^{c, n, 0}\right|_{\partial \Omega_{I}^{c}}=\left.\hat{\mathbf{u}}^{c, n, 0}\right|_{\partial \Omega_{I}^{c}}+\left.\delta \hat{\mathbf{n}}\right|_{\partial \Omega_{I}^{c}} .
\end{aligned}
$$

In (39), we compute the sub-timestep solution for $\Omega^{f}$, sequentially from $i=1 \ldots \eta$, and in (40), we (concurrently) compute the solution in $\Omega^{c}$ at time $t^{n}$. 
2. Once the predictor step is complete, $q=1 \ldots Q$ corrector iterations are done.

- Interpolate the interdomain boundary data:

$$
\begin{aligned}
\left.\hat{\mathbf{u}}^{f, n-1+\frac{i}{\eta}, q}\right|_{\partial \Omega_{I}^{f}} & =\mathcal{I}\left(\zeta_{i 1} \mathbf{u}^{c, n, q-1}+\zeta_{i 2} \mathbf{u}^{c, n-1, Q}+\zeta_{i 3} \mathbf{u}^{c, n-2, Q}\right), \quad i=1 \ldots \eta \\
\left.\hat{\mathbf{u}}^{c, n, q}\right|_{\partial \Omega_{I}^{c}} & =\mathcal{I}\left(\mathbf{u}^{f, n, q-1}\right) .
\end{aligned}
$$

- Unsteady Stokes solve for the $i=1 \ldots \eta$ sub-timesteps of $\Omega^{f}$ :

$$
\begin{aligned}
\dot{\mathbf{u}}^{f, n-1+\frac{i}{\eta}} & =-\sum_{j=1}^{k} \beta_{j} \mathbf{u}^{f, n-1-\frac{j-i}{\eta}, Q}+\Delta t_{f} \sum_{j=1}^{k} \alpha_{j} \mathbf{f}_{e}^{f, n-1-\frac{j-i}{\eta}, Q}, \\
\mathbf{S} \phi^{f, n-1+\frac{i}{\eta}, q} & =\mathbf{r}^{f, n-1+\frac{i}{\eta}, q},\left.\mathbf{u}^{f, n-1+\frac{i}{\eta}, q}\right|_{\partial \Omega_{I}^{f}}=\left.\hat{\mathbf{u}}^{f, n-1+\frac{i}{\eta}, q}\right|_{\partial \Omega_{I}^{f}}+\left.\delta \hat{\mathbf{n}}\right|_{\partial \Omega_{I}^{f}} .
\end{aligned}
$$

- Unsteady Stokes solve for the only timestep of $\Omega^{c}$ :

$$
\mathbf{S} \phi^{c, n, q}=\mathbf{r}^{c, n, q},\left.\quad \mathbf{u}^{c, n, q}\right|_{\partial \Omega_{I}^{c}}=\left.\hat{\mathbf{u}}^{c, n, q}\right|_{\partial \Omega_{I}^{c}}+\left.\delta \hat{\mathbf{n}}\right|_{\partial \Omega_{I}^{c}}
$$

Using the high-order multirate timestepping strategy in $(37)-(44)$, the solution to the incompressible Navier-Stokes equations can be advanced in two overlapping grids for an arbitrary timestep ratio.

\subsection{Multirate Timestepping in $S>2$ Overlapping Domains}

With the multirate timestepping scheme that we have described, it is straightforward to scale this method to an arbitrary number of domains. Figure 7 shows an example of a schematic with time-levels for MTS in $s=1 \ldots S$ subdomains.

For notational purposes, we will use $\Omega^{c}$ to represent the subdomain with slowest timescales. With each subdomain, we associate the timestep ratio with respect to the timestep size of $\Omega^{c}$ :

$$
\eta_{s}:=\frac{\Delta t_{c}}{\Delta t_{s}}
$$

such that $\eta_{c}=1$ and $\eta_{s}>1$ for $s \neq c$. For the example in Fig. 7, $c=1$ and the timestep ratios for different subdomains are $\eta_{1}=1, \eta_{2}=\Delta t_{1} / \Delta t_{2}=4$ and $\eta_{3}=\Delta t_{1} / \Delta t_{3}=6$.

Using (45) and assuming that the interdomain boundary data for points on $\partial \Omega_{I}^{s}$ is interpolated from $\Omega^{r}$, the MTS for arbitrary number of subdomains $(s=1 \ldots S)$ is:

1. Solve the unsteady Stokes problem for $i=1 \ldots \eta_{s}$ sub-timesteps.

- Interpolate the interdomain boundary data (Section 2.4.1):

$$
\left.\hat{\mathbf{u}}^{n-1+\frac{i}{\eta_{s}}}\right|_{\partial \Omega_{I}^{s}}=\mathcal{I}\left(\sum_{j=1}^{m} \gamma_{i j} \mathbf{u}^{r, n-1-\frac{j-1}{\eta_{r}}, Q}\right)
$$




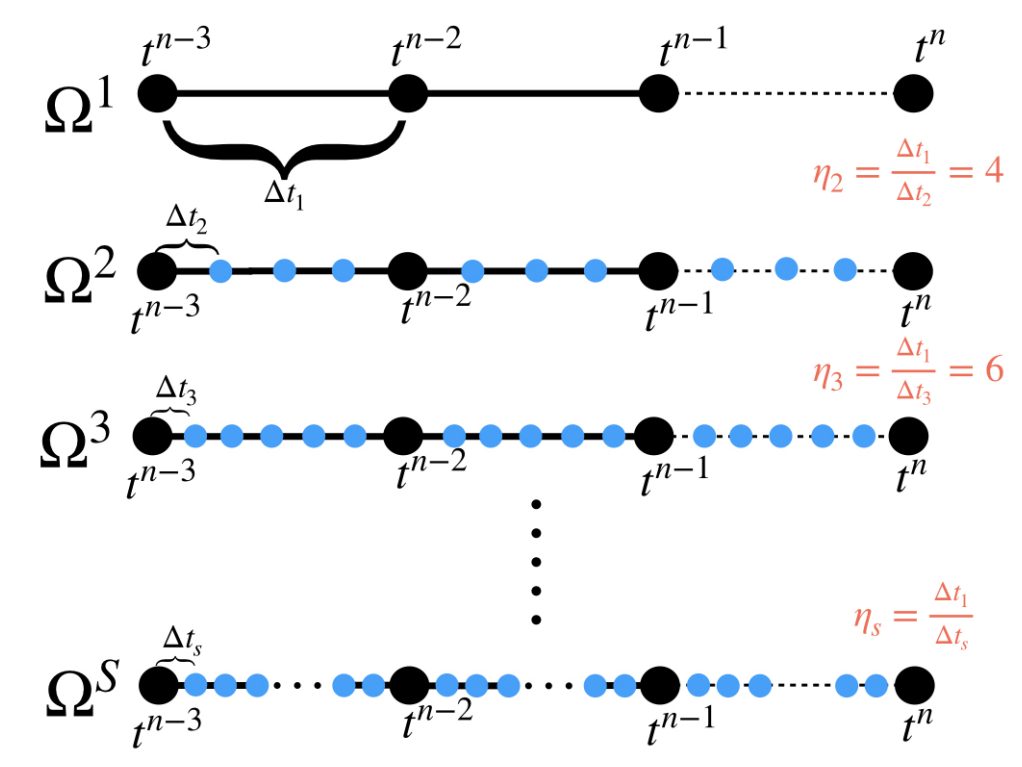

Figure 7: Schematic showing discrete time-levels for the multirate timestepping in an arbitrary number of subdomains.

- Unsteady Stokes solve in each subdomain (Section 2.3) for the $i=1 \ldots \eta_{s}$ sub-timesteps during the predictor stage with flux-corrected boundary data on $\partial \Omega_{I}^{s}$ (Section 2.4.2):

$$
\begin{aligned}
\dot{\mathbf{u}}^{s, n-1+\frac{i}{\eta_{s}}} & =-\sum_{j=1}^{k} \beta_{j} \mathbf{u}^{s, n-1-\frac{j-i}{\eta_{s}}, Q}+\Delta t_{s} \sum_{j=1}^{k} \alpha_{j} \mathbf{f}_{e}^{s, n-1-\frac{j-i}{\eta_{s}}, Q}, \\
\mathbf{S} \boldsymbol{\phi}^{s, n-1+\frac{i}{\eta_{s}}, 0} & =\mathbf{r}^{s, n-1+\frac{i}{\eta_{s}}, 0},\left.\mathbf{u}^{n-1+\frac{i}{\eta_{s}}}\right|_{\partial \Omega_{I}^{s}}=\left.\hat{\mathbf{u}}^{n-1+\frac{i}{\eta_{s}}}\right|_{\partial \Omega_{I}^{s}}+\left.\delta \hat{\mathbf{n}}\right|_{\partial \Omega_{I}^{s}}
\end{aligned}
$$

2. Once the predictor step is complete, do $q=1 \ldots Q$ corrector iterations.

- Interpolate the interdomain boundary data (Section 2.4.1):

$$
\left.\hat{\mathbf{u}}^{n-1+\frac{i}{\eta_{s}}}\right|_{\partial \Omega_{I}^{s}}=\mathcal{I}\left(\zeta_{i 1} \mathbf{u}^{r, n, q-1}+\zeta_{i 2} \mathbf{u}^{r, n-1, Q}+\zeta_{i 3} \mathbf{u}^{c, n-1-\frac{1}{\eta_{r}}, Q}\right)
$$

- Unsteady Stokes solve in each subdomain (Section 2.3) for the $i=1 \ldots \eta_{s}$ sub-timesteps during each of the $Q$ corrector iterations. Each unsteady Stokes solve uses the fluxcorrected boundary data on $\partial \Omega_{I}^{s}$ (Section 2.4.2):

$$
\begin{aligned}
\dot{\mathbf{u}}^{s, n-1+\frac{i}{\eta_{s}}} & =-\sum_{j=1}^{k} \beta_{j} \mathbf{u}^{s, n-1-\frac{j-i}{\eta_{s}}, Q}+\Delta t_{s} \sum_{j=1}^{k} \alpha_{j} \mathbf{f}_{e}^{s, n-1-\frac{j-i}{\eta_{s}}, Q}, \\
\mathbf{S} \phi^{s, n-1+\frac{i}{\eta_{s}}, q} & =\mathbf{r}^{s, n-1+\frac{i}{\eta_{s}}, q},\left.\mathbf{u}^{n-1+\frac{i}{\eta_{s}}}\right|_{\partial \Omega_{I}^{s}}=\left.\hat{\mathbf{u}}^{n-1+\frac{i}{\eta_{s}}}\right|_{\partial \Omega_{I}^{s}}+\left.\delta \hat{\mathbf{n}}\right|_{\partial \Omega_{I}^{s}} .
\end{aligned}
$$




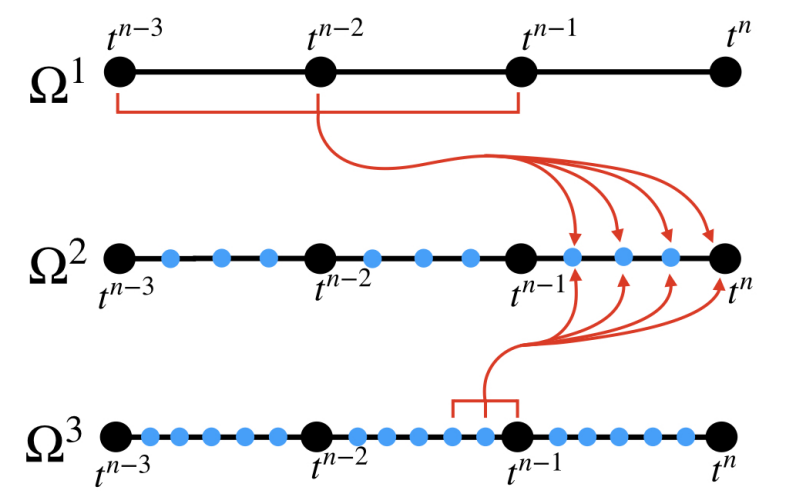

(a) Interdomain boundary data dependency for $\Omega^{2}$ at the predictor step.

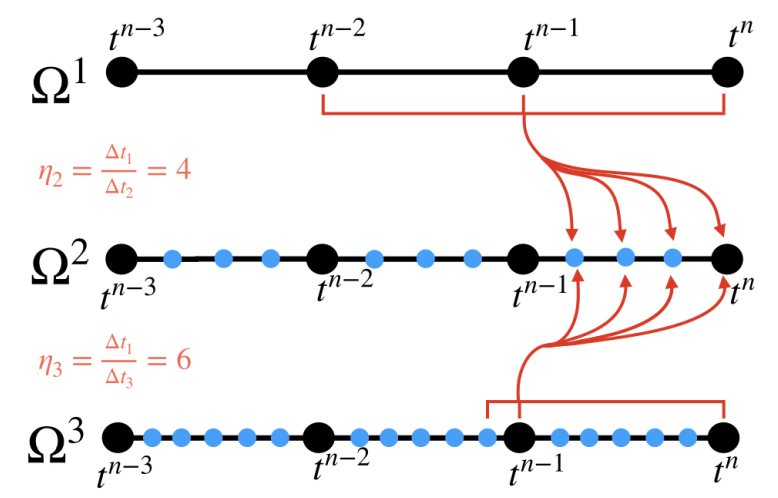

(b) Interdomain boundary data dependency for $\Omega^{2}$ at the corrector step.

Figure 8: Schematic showing interdomain boundary data dependency for $\Omega^{2}$ from $\Omega^{1}$ and $\Omega^{3}$.

Equations (46)- (49) describe the MTS method for solving the INSE in an arbitrary number of overlapping grids with an arbitrary integer timestep ratio. From an implementation perspective, since different interdomain boundary points in a grid can overlap different grids, the coefficients $\gamma_{i j}$ and $\zeta_{i j}$ are computed for each point based on the timestep size of the donor subdomain $\left(\Omega^{r}\right)$. Note that for cases where all the meshes are fixed and the timestep size is constant in each subdomain, these coefficients thus need to be computed only at the beginning of the calculation.

Figure 8 shows an example of the interdomain boundary data dependency for the schematic shown in Fig. 7. Here, we assume that the gridpoints on $\partial \Omega_{I}^{2}$ overlap $\Omega^{1}$ or $\Omega^{3}$. Assuming that the solution is know up to time $t^{n-1}$, the boundary data for $\partial \Omega_{I}^{2}$ is extrapolated for the predictor step using $\phi^{r, n-1, Q}, \phi^{r, n-1-\frac{1}{\eta_{r}}, Q}$, and $\phi^{r, n-1-\frac{2}{\eta_{r}}, Q}$, where $r=1$ or 2 . Similarly, the boundary data for $\partial \Omega_{I}^{2}$ for the corrector steps $(q=1 \ldots Q)$ is extrapolated from the most recent Schwarz iteration $\phi^{r, n, q-1}$ and the converged solutions $\phi^{r, n-1, Q}$ and $\phi^{r, n-1-\frac{1}{\eta_{r}}, Q}$.

We note that unlike the singlerate timestepping scheme where only 1 interpolation is re- 
quired at each predictor and corrector iteration, the multirate timestepping scheme requires $m$ interpolations at the beginning of each predictor step and 1 interpolation at the beginning of each corrector iteration. Thus, for $m$-th order temporal accuracy with an example corresponding to timestep ratio $\eta$, the STS scheme requires a total of $(Q+1) \eta$ interpolations $(Q+1$ at each sub-timestep) and the MTS scheme requires a total of $m+Q$ interpolations. Consequently, the MTS typically requires fewer interpolations in comparison to the STS scheme. In Section 4.3, we will use the example of a thermally-buoyant plume to compare the total time to solution between the STS and MTS scheme with $\eta=5$.

\subsection{Stability Considerations}

An underlying assumption of the MTS scheme is that each subdomain has a timestep size that satisfies its CFL stability criterion (2). This requirement ensures intradomain stability, i.e., the unsteady Stokes solve for time-advancing the solution of the INSE is stable in each subdomain. Interdomain stability, however, is similar to the singlerate timestepping scheme and depends on the order of extrapolation $(m)$ used for interdomain boundary data and the number of Schwarz iterations $(Q)$ used at each timestep.

Peet and Fischer [36] have analyzed the stability of the singlerate timestepping scheme using an FD-based framework to show than $Q=1-3$ is sufficient from a stability and accuracy point of view when $m>1$. We have extended their method to analyze the stability of the MTS scheme for $S=2$ [44]. There are two important results that have come forth from this analysis. First, we note that the MTS scheme requires at-least one Schwarz iteration for stability when $m>1$, depending on the timestep ratio. For $m=1$, however, $Q=0$ is sufficient for stability regardless of the timestep ratio. Second, we observe that for STS, odd values of $Q$ are more stable than even values of $Q$. In contrast, for MTS with $\eta=2$, even- $Q$ is more stable than odd- $Q$. This odd-even stability pattern goes away for large timestep ratios $(\eta \geq 4)$. The results that we have observed in our stability analysis are similar to observations about predictor-corrector methods by Stetter [45] and Love et al. [46].

In Section 4.2, we will demonstrate that $Q=0, m=1$ is sufficient from an accuracy point of view when the subdomains overlap away from the region of interest. This observation is in agreement with the results in [1] that show that for the STS-based Schwarz-SEM framework, the noniterated case $(Q=0)$ provides a fast and sufficiently accurate pathway for basic statistics (e.g., mean and rms) of turbulence in complex domains.

\section{Results}

In this section, we demonstrate the effectiveness of the MTS-based strategy for solving the incompressible Navier-Stokes equations with two different examples. In the first example, we use a problem with a known exact solution to demonstrate the spatial and temporal convergence of the MTS method. Next, we use this method to model a buoyant thermal plume in a stratified environment, where the INSE is solved on overlapping grids with $\eta=$ 100.

\subsection{Exact Solution for Decaying Vortices}

Our first example is due to Walsh [47], who derived a family of exact Navier-Stokes

eigenfunctions that can be used to test spatial and temporal convergence of discretizations 
of the INSE. The eigenfunctions are linear combinations of $\cos (p x) \cos (q y), \sin (p x) \cos (q y)$, $\cos (p x) \sin (q y)$, and $\sin (p x) \sin (q y)$, for all integer pairs $(p, q)$ satisfying $\lambda=-\left(p^{2}+q^{2}\right)$. Taking as an initial condition the eigenfunction $\hat{\mathbf{u}}=\left(-\psi_{y}, \psi_{x}\right)$, a solution to the INSE is $\mathbf{u}=e^{\nu \lambda t} \hat{\mathbf{u}}(\mathbf{x})$. Here, $\psi$ is the streamfunction resulting from the linear combinations of eigenfunctions. Interesting long-time solutions can be realized by adding a relatively highspeed mean flow $\mathbf{u}_{0}$ to the eigenfunction, in which case the solution is $\mathbf{u}_{\text {exact }}=e^{\nu \lambda t} \hat{\mathbf{u}}\left[\mathbf{x}-\mathbf{u}_{0} t\right]$, where the brackets imply that the argument is modulo $2 \pi$ in $x$ and $y$. As a result, this problem lets us test our algorithm in the advection-dominated limit. (The alternative of simply decreasing $\nu$ can yield to chaotic solutions because the exact eigenfunctions are not stable solutions to the INSE at elevated Reynolds numbers.)

Here, we model a periodic domain $\Omega:=[0,2 \pi]^{2}$ using three overlapping meshes that are illustrated Fig. 9(a). A doubly-periodic background mesh $\left(\Omega^{1}\right.$ with $\left.E=240\right)$ has a square hole in the center that is covered with a pair of circular meshes $\left(\Omega^{2}\right.$ with $E=96$ and $\Omega^{3}$ with $E=140)$. The individual meshes are shown along with their interdomain boundaries in Fig. 9 (b). The flow parameters are $\nu=0.05, \mathbf{u}_{0}=(1,0.3), \psi=(1 / 5) \sin (5 y)+(1 / 5) \cos (5 x)-$ $(1 / 4) \sin (3 x) \sin (4 y)$, and $\lambda=-25$.

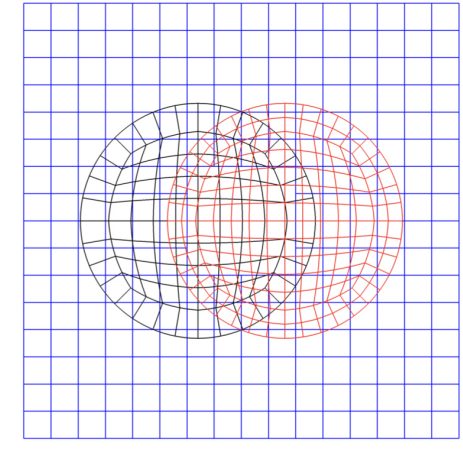

(a)

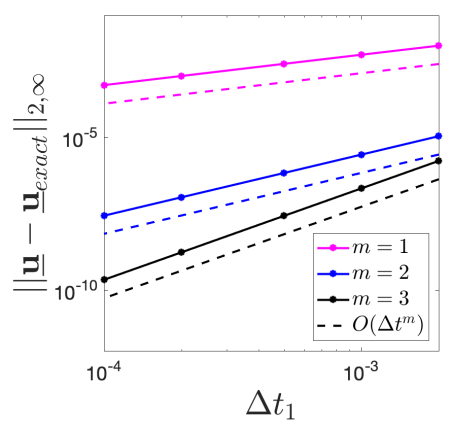

(c)

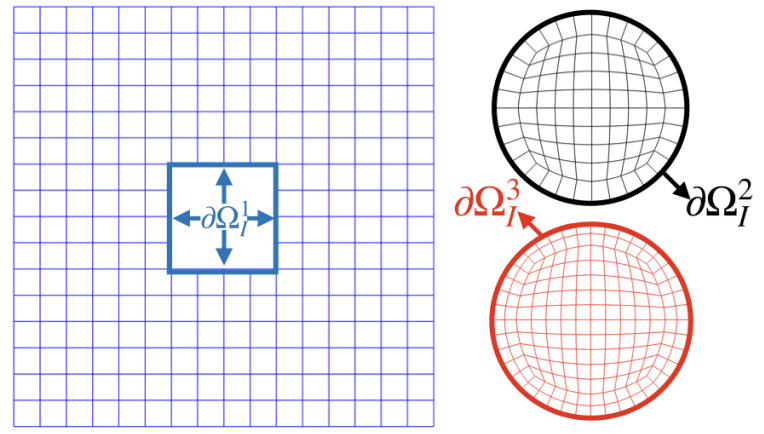

(b)

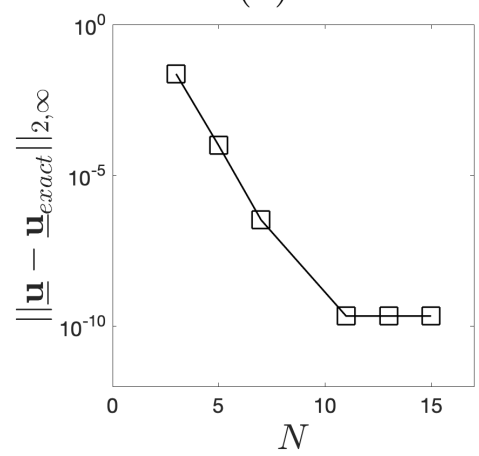

(d)

Figure 9: (a) Overlapping and (b) individual spectral element meshes for the doubly-periodic domain, (c) temporal and (d) spatial convergence of the error in the solution.

To demonstrate temporal convergence of the MTS-based method, the flow is integrated up to time $T_{f}=1$ convective time units (CTU) at different $\Delta t$ for extrapolation order $m=1$, 2 , and 3. To ensure stability of the predictor-corrector approach for multirate timestepping, we set $Q=0$ for $m=1, Q=1$ for $m=2$, and $Q=3$ for $m=3$. The interpolation order during the correction iterations is set to $\tilde{m}=\max (1, m-1)$. Additionally, because 
of the difference in the size of the elements in the three meshes, the timestep ratio is set to $\Delta t_{1} / \Delta t_{2}=2$ and $\Delta t_{1} / \Delta t_{3}=3$ to keep the CFL similar for the three subdomains. The polynomial order is $N=13$ for this convergence study and the BDF3/EXT3 scheme is used for all the results presented here.

Figure 9(c) shows that the MTS-based method maintains the temporal convergence of the underlying SEM solver. Here, the error is computed as $\underline{\mathbf{e}}=\underline{\mathbf{u}}-\underline{\mathbf{u}}_{\text {exact }}$, and the norm is the 2-norm of the point-wise maximum of the vector field, i.e., $\|\underline{\mathbf{e}}\|_{2, \infty}:=\|\tilde{\mathbf{e}}\|_{2}$, where $\tilde{\mathbf{e}}=\left[\left\|\underline{e}_{1}\right\|_{\infty},\left\|\underline{e}_{2}\right\|_{\infty}\right]$. For each $m=1,2$ and 3 , we observe that the error between the numerical solution and the exact solution decreases as $O\left(\Delta t^{m}\right)$.

Similarly, for spatial convergence, the flow is integrated up to time $T_{f}=1$ convective time units (CTU) at different $N$. The timestep size is fixed to $\Delta t_{1}=10^{-4}$ and $Q=3$ corrector iterations are used at each timestep with third-order extrapolation $(m=3)$ for the interdomain boundary data. Figure 9(d) shows the exponential convergence of the solution obtained using MTS method with change in $N$. The temporal and spatial convergence results presented here demonstrate that the MTS method presented in this paper maintains the convergence properties of the underlying SEM solver.

\subsection{Buoyant Thermal Plume}

Buoyant plumes arise in a variety of industrial and environmental flow problems such as deepwater blowouts [48], volcanoes [49] and hydrothermal vents [50, and they have been the subject of several experimental and computational studies (e.g., [7, 51, 52, 53, 54]). As noted in Section 1, plumes often feature significant scale disparity; the high speed, highly turbulent, flow near the plume or jet exit requires fine scale meshes and correspondingly small timestep sizes in that region, whereas the far-field flow is typically relatively quiescent, with larger-scale and slower turbulent eddies. Thus, buoyant plumes (and even non-buoyant jets) are ideal candidates for discretizations that are multi-resolution both in space and time. This point is emphasized by the example of Fig. 1, which shows a multidomain spatial discretrization where the near-field CFL is about 100 times larger than the far-field CFL when using an STS.

To explore the potential of our Schwarz-MTS coupling for this class of problems, we consider a singlephase thermally-buoyant plume in a stratified environment. In this example, we assume that there is a reference density $\left(\rho_{r}\right)$ and a reference temperature $\left(T_{r}\right)$, with respect to which the density varies in the domain as $\rho=\rho_{r}\left(1-\gamma\left(T-T_{r}\right)\right)$, where $\gamma$ is the thermal expansion coefficient of the fluid. We also assume that the temperature in the domain, which is a solution of (5), can be described as $T(\mathbf{x}, t)=\theta(\mathbf{x}, t)+T_{r}+\Gamma z$, where $\theta$ is the perturbation with respect to the unperturbed environment temperature, $T_{e}$, which varies linearly with a slope of $\Gamma$ as $T_{e}=T_{r}+\Gamma z$. Here, $z$ is the direction in which the fluid is stratified, which is always in opposite direction to the gravitational acceleration for stable stratification.

The effect of variation in density due to the temperature difference in the fluid and the transport of $\theta$ in the domain is modeled using the Boussinesq approximation [55]. These 
assumptions lead to a system of the form

$$
\begin{aligned}
\frac{\partial \mathbf{u}}{\partial t}+\mathbf{u} \cdot \nabla \mathbf{u} & =-\nabla p+\frac{1}{R e} \nabla^{2} \mathbf{u}+\operatorname{Ri\theta } \hat{k} \\
\nabla \cdot \mathbf{u} & =0 \\
\frac{\partial \theta}{\partial t}+\mathbf{u} \cdot \nabla \theta & =\frac{1}{P e} \nabla^{2} \theta-\mathbf{u} \cdot \hat{k}
\end{aligned}
$$

which we solve using the Schwarz-SEM framework. In $50, R i=g /\left(B_{o}^{1 / 4} N_{b}^{5 / 4}\right)$ is the Richardson number that depends on the acceleration due to gravity $(g)$, inlet buoyancy flux $\left(B_{0}\right)$, and the buoyancy frequency $\left(N_{b}\right)$ (also known as the Brunt-Väisälä frequency). The velocity, time, length, pressure, and temperature scales used for nondimensionalization are $U_{0}=\left(B_{0} N_{b}\right)^{1 / 4}, t_{0}=1 / N_{b}, L_{0}=\left(B_{0} / N_{b}^{3}\right)^{1 / 4}, p_{0}=\rho_{r} U_{0}^{2}$, and $T_{0}=\Gamma L_{0}=\left(B_{0} N_{b}^{5}\right)^{1 / 4} /(g \gamma)$, where $g$ is the acceleration due to gravity. We assume that the reference density is $\rho_{r}=1$ and the reference temperature is $T_{r}=0$. The temperature solution $T(\mathbf{x}, t)$ can be obtained by substituting $T(\mathbf{x}, t)=\theta(\mathbf{x}, t)+T_{r}+\Gamma z$.

Here, we follow [7] and specify $B_{0}=5 \times 10^{-6} m^{4} s^{-3}, N_{b}=0.1 s^{-1}$. The linear scaling for density is set to $\gamma=2 \times 10^{-4} K^{-1}$, and for temperature to $\Gamma=5.1 \mathrm{~m}^{-1}$. Double diffusion effects are ignored, and thus $\nu=\alpha=10^{-6} \mathrm{~m}^{2} \mathrm{~s}^{-1}$, which leads to Prandtl number $\operatorname{Pr}=1$. The Reynolds number $\left(U_{0} L_{0} / \nu\right)$ and Peclet number of the flow is about 7100 , and the Richardson number is 3700. The reader is referred to Fabregat et al. [7] for a detailed derivation of the governing equations for this example.

To validate the MTS scheme, we are interested in accurately determining three key plume parameters (illustrated in Fig. 10) and comparing these results from MTS with the monodomain SEM framework and [7]:

- Maximum height $z_{\max }$ - the maximum height at which the axial velocity of the plume vanishes.

- Trapping height $z_{t h}$ - the height of the centerline of the outgoing gravity current.

- Equilibrium height $z_{e q}$ - the height at which the plume becomes neutrally buoyant.

Note that the results presented in [7] were also obtained using the monodomain SEM framework. The only difference in the problem setup of [7] from the ongoing calculations is that Fabregat et al. do not model the recycling-pipe attached at the bottom of the cylindrical tank (Fig. 1), and instead applied inhomogeneous Dirichlet condition (defined an inlet velocity) directly at the bottom of the tank. The recycling-pipe inlet was implemented in the current setup to allow for fully developed turbulent inflow in the plume. Though, difference in the inlet setup between [7] and the current study will not have appreciable affect on the three parameters defined above, as every correctly simulated stable turbulent buoyant plume converges to the asymptotic solution derived by Morton-Taylor-Turner [56].

\subsubsection{Results}

Figure 1(b,c)-(d) shows the spectral element meshes that were used for the monodomain and Schwarz-SEM calculations, respectively. The conforming mesh for monodomain SEM has 76,600 elements, and the overlapping spectral element meshes have a total of 71,040 


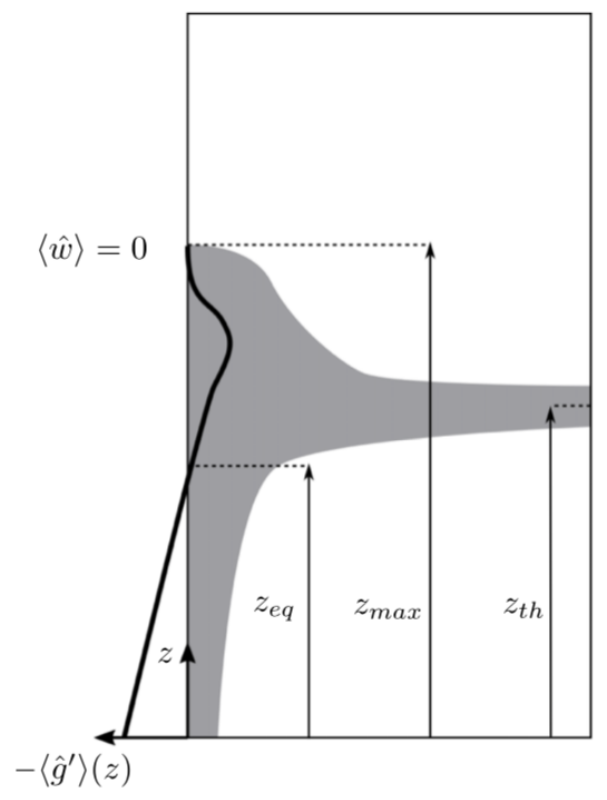

Figure 10: Schematic of a singlephase plume indicating the maximum plume height $\left(z_{\max }\right)$, trapping height $\left(z_{t h}\right)$ and equilibrium height $\left(z_{e q}\right)$. Image taken from [7].

elements. $E_{f}=55,480$ elements for the dense inner grid $\left(\Omega^{f}\right)$ and $E_{c}=15,560$ elements in the coarse outer grid $\left(\Omega^{c}\right)$. The total element count is lower for the Schwarz-SEM framework because the overlapping meshes are nonconforming with the outer mesh much coarser as compared to the inner-mesh.

Using the multirate timestepping method described in Section 3, two different timestep ratio are used for the Schwarz-SEM framework; $\eta=5$ and $\eta=100$. Since the subdomain with slower time-scales has to take many fewer timesteps in the MTS-based scheme, we use fewer MPI ranks for the outer domain in comparison to the MPI ranks needed for the STS-based scheme. The timing analysis for the MTS-based scheme has been presented in Section 4.3 .
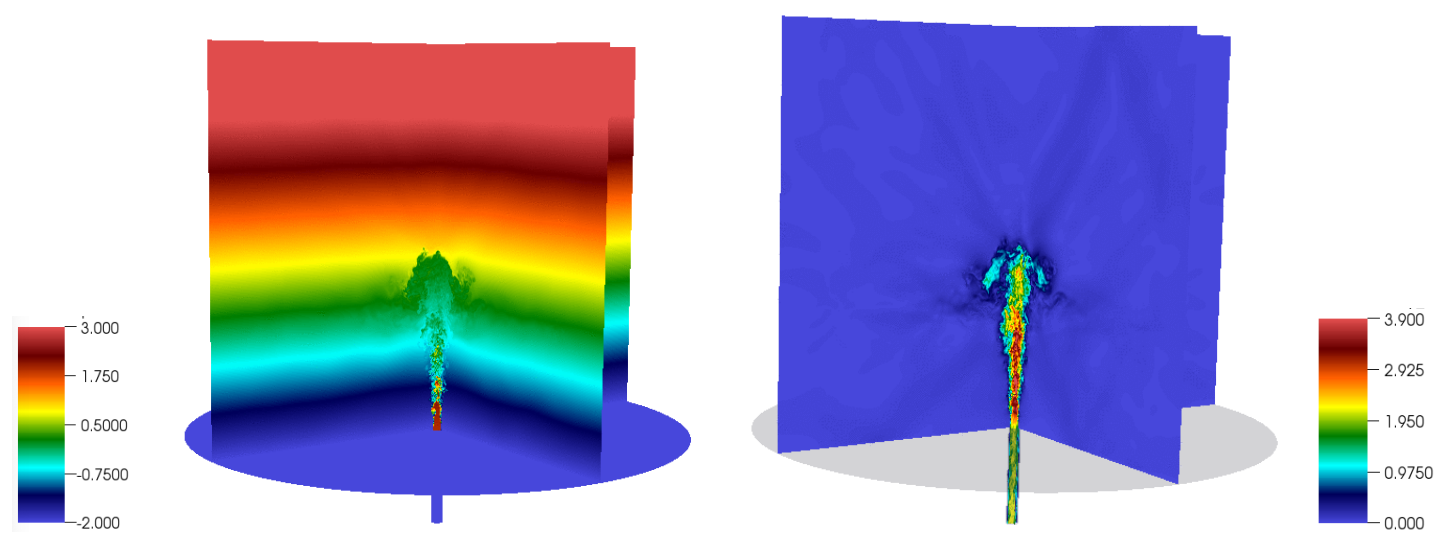

Figure 11: Snapshot of instantaneous (a) temperature and (b) velocity magnitude solution from the overlapping grid calculation with $\eta=100$. 


\begin{tabular}{|r|c|c|c|}
\hline & $z_{\max }$ & $z_{e q}$ & $z_{t h}$ \\
\hline \hline Fabregat et al. & 4.5 & 2.85 & 3.11 \\
\hline Monodomain SEM & 4.51 & 2.85 & 3.15 \\
\hline Schwarz-SEM $\eta=5$ & 4.56 & 2.85 & 3.15 \\
\hline Schwarz-SEM $\eta=100$ & 4.46 & 2.85 & 3.19 \\
\hline
\end{tabular}

Table 1: $z_{\max }, z_{e q}$ and $z_{t h}$ obtained from the Schwarz-SEM framework, the current monodomain calculation, and the monodomain calculation by Fabregat [7].

Following the monodomain calculation, the polynomial order is set to $N=7$ for the overlapping grid calculation. Since the overlap region is away from the area of interest, we set $Q=0$ with $m=1$, and the flow statistics are temporally-averaged over more than $30 \mathrm{CTU}$. Figure 11 shows a snapshot of the instantaneous temperature and velocity magnitude solution from the overlapping grid calculation with $\eta=100$. Figure 12 shows the temporally and spatially averaged (azimuthally averaged) velocity magnitude, temperature perturbation, and turbulent kinetic energy (TKE) contours, respectively, for the overlapping grid $(\eta=5$ and 100) and monodomain calculations. We observe that the Schwarz-SEM framework gives good comparison with the monodomain SEM calculation even when the timestep ratio is 100.

Using the temporally and spatially averaged axial velocity $\left(W_{\text {mean }}\right)$ and temperature perturbation $\left(\theta_{\text {mean }}\right)$ along the plume centerline, we can obtain the maximum height of the plume $\left(z_{\max }\right)$ and equilibrium height $\left(z_{e q}\right)$. We can also use the TKE plots to obtain the plume trapping height $\left(z_{t h}\right)$. The line plots comparing $W_{\text {mean }}$ and $\theta_{\text {mean }}$ are shown in Fig. 13, and show good comparison between the three cases considered here.

Table 1 compares the maximum plume height $\left(z_{\max }\right)$, equilibrium height $\left(z_{e q}\right)$, and trapping height $\left(z_{t h}\right)$ for the Schwarz-SEM results with monodomain calculations and the monodomain SEM results of Fabregat et al. [7]. The maximum difference between the SchwarzSEM calculations and monodomain calculation for the three parameters of interest is $1.2 \%$ (for $z_{\max }$ ). The trapping height $\left(z_{t h}\right)$ for our monodomain calculation is different from Fabregat et al., and that is expected as the flow was not fully turbulent in the plume in [7].

The results presented in this section demonstrate the effectiveness of the MTS method in simulating complex turbulent flow and heat transfer phenomena. This MTS method will be used for understanding the behavior of singlephase and multiphase rotating plumes in a cross-flow. This target problem is intractable with a monodomain SEM framework because a conforming mesh leads to a high element count, an issue that overlapping grids help us circumvent.

\subsection{Timing Comparison between STS and MTS Method}

With multirate timestepping, the subdomain with faster time-scales $\left(\Omega^{f}\right)$ uses a smaller timestep size with more timesteps in comparison to the subdomain with slower time-scales $\left(\Omega^{c}\right)$.

Here, we use the thermally-buoyant problem with $\eta=5$ to demonstrate that multirate timestepping reduces the computational cost in comparison to the corresponding singlerate timestepping-based $(\eta=1)$ calculation. For this example, $E_{f}=55,480$ elements for the 

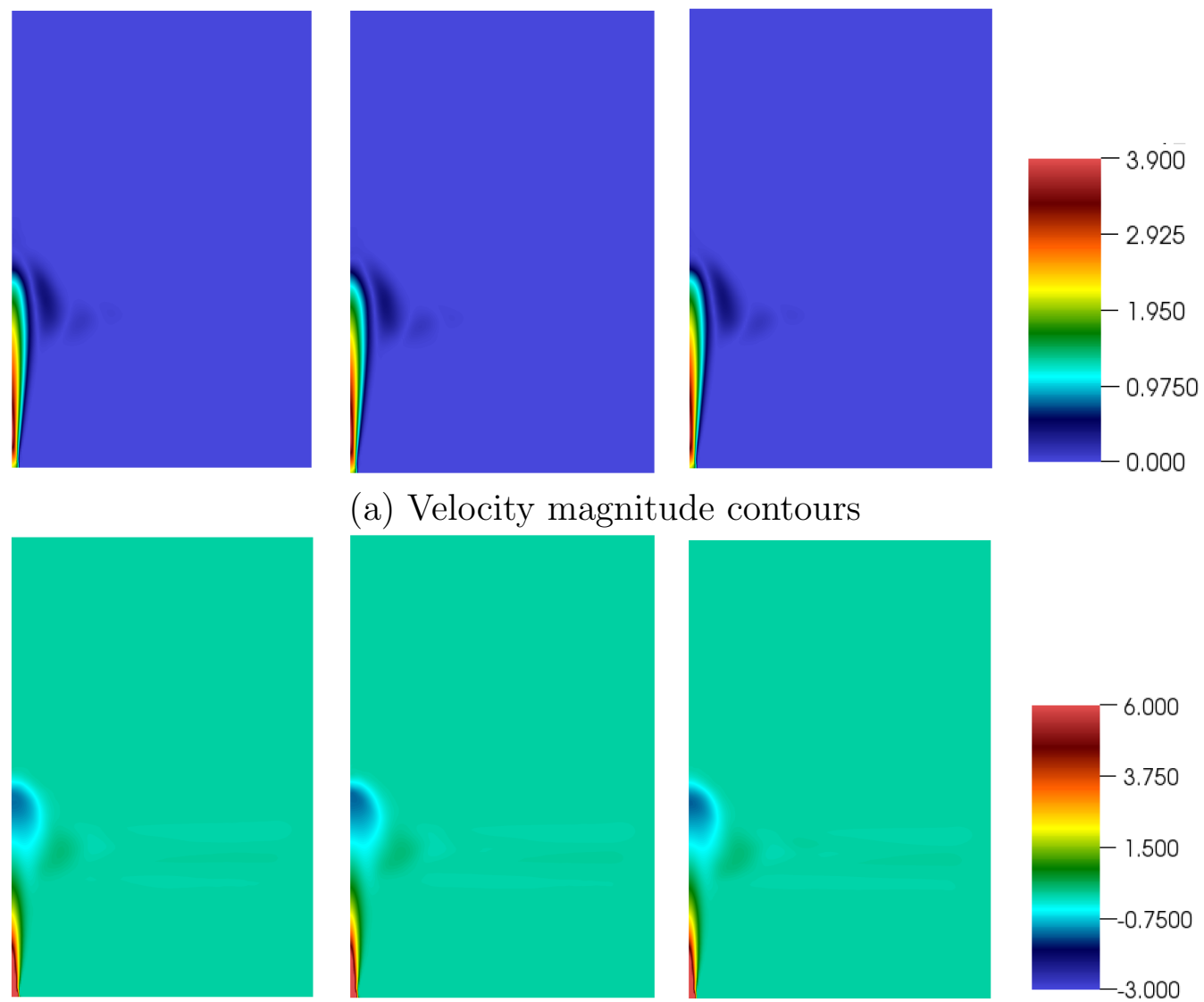

(a) Velocity magnitude contours
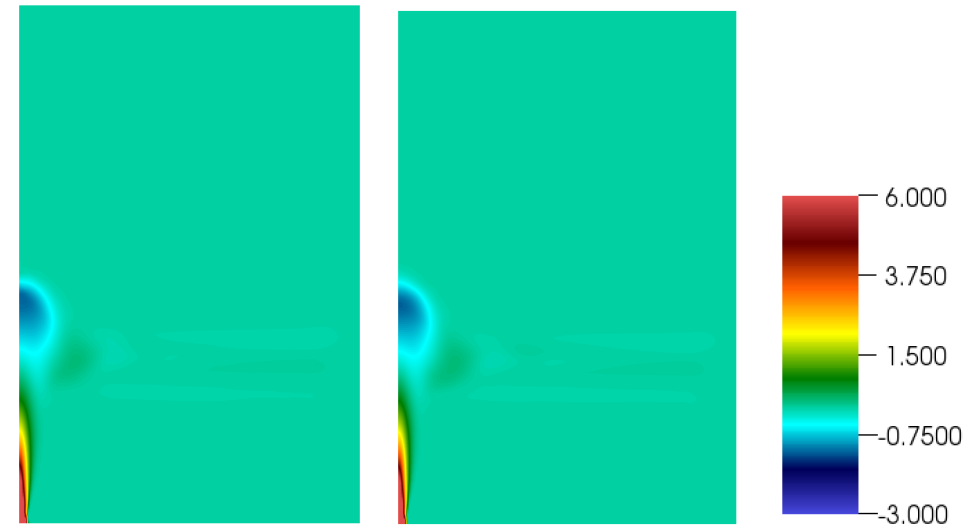

(b) Temperature perturbation contours
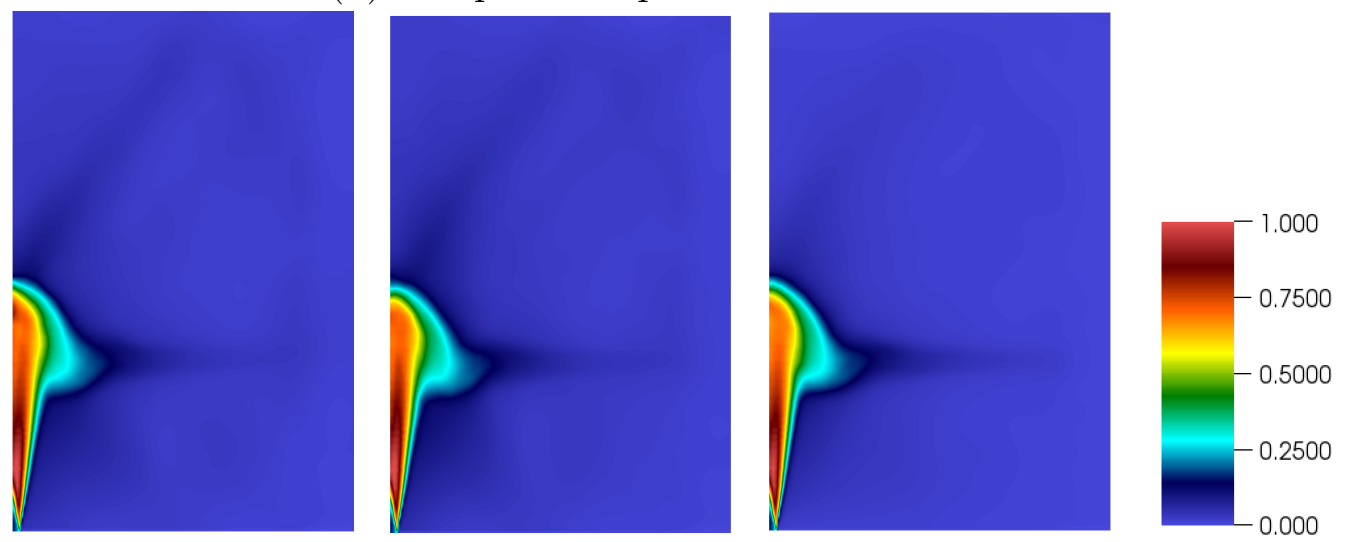

(c) Turbulent kinetic energy contours

Figure 12: Temporally and spatially averaged (top to bottom) (a) velocity magnitude, (b) temperature perturbation, and (c) turbulent kinetic energy for the Schwarz-SEM calculations with overlapping grids using (left) $\eta=5$ and (center) $\eta=100$, and (right) the monodomain SEM calculation. 

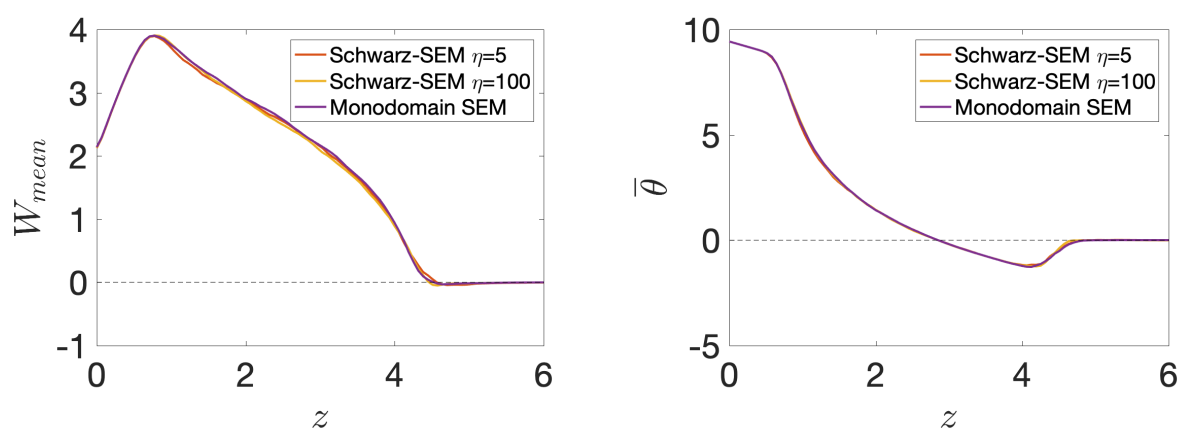

Figure 13: Temporally and spatially averaged (left) axial velocity $\left(W_{\text {mean }}\right)$ and (right) temperature perturbation $\left(\theta_{\text {mean }}\right)$ along the plume centerline.

dense inner grid $\left(\Omega^{f}\right)$ and $E_{c}=15,560$ elements in the coarse outer grid $\left(\Omega^{c}\right)$. For overlapping subdomains, ideally one would partition the domain in parallel such that the time to solution per timestep $\left(T_{\text {step }}\right)$ is similar for each subdomain. For the singlerate timestepping scheme, we typically choose the number of MPI ranks $(P)$ for each subdomain using

$$
\frac{P_{c}}{P_{f}} \approx \frac{E_{c}}{E_{f}}
$$

where $P_{f}$ and $P_{c}$ are the number of MPI ranks use to partition $\Omega^{f}$ and $\Omega^{c}$, respectively. Based on $E_{c}$ and $E_{f}$ for this example, we set $P_{c} \approx P_{f} / 4$. For the multirate scheme, however, since $\Omega^{c}$ has many times fewer steps as compared to the $\Omega^{f}$, the number of MPI ranks for $\Omega^{c}$ can be reduced even further.

Figure 14 compares how the mean time to solution per timestep $\left(T_{\text {step }}\right)$ varies with $P_{c}$ for the singlerate and multirate timestepping scheme, while keeping $P_{f}$ fixed at $4096 \mathrm{MPI}$ ranks. These calculations were done with $m=1, Q=0$, and $N=7$. The time per timestep was obtained for multirate timestepping scheme by monitoring the mean time taken by $\Omega^{f}$ for each sub-timestep, which is equivalent to a single timestep in the singlerate timestepping scheme. The timestep size was kept same for $\Omega^{f}$ for the multirate and singlerate timestepping scheme, to ensure fair comparison. The numerical experiments discussed here were done on Cetus, an IBM Blue Gene/Q at the Argonne Leadership Computing Facility

The singlerate timestepping scheme is most efficient when $P_{c}=1024=P_{f} / 4$, which is in agreement with $(53)$ since $E_{c} \approx E_{f} / 4$. As $P_{c}$ is decreased, the time to solution increases as expected. We also notice that increase in $P_{c}$ beyond 1024 does not change or decrease the time to solution, which is due to $T_{\text {step }}$ being limited by $\Omega^{f}$ once $P_{c}>P_{f} / 4$. In contrast to the singlerate scheme, since $\Omega^{c}$ has to take fewer timesteps with the multirate timestepping scheme, $P_{c}=P_{f} / 16$ is as effective as $P_{c}=P_{f} / 4$. $P_{c}$ cannot be reduced further because of the constraint on maximum memory that can be allocated on each MPI rank on Cetus. Additionally, we see that the multirate timestepping scheme does better than the singlerate timestepping scheme for equivalent number of MPI ranks when $P_{c} \geq 1024$. This difference is because in the MTS-based scheme, the interdomain boundary data is exchanged fewer times between the different domains and each subdomain only needs to re-evaluate extrapolation/interpolation coefficient at each sub-timestep. In contrast, STS-based scheme requires 


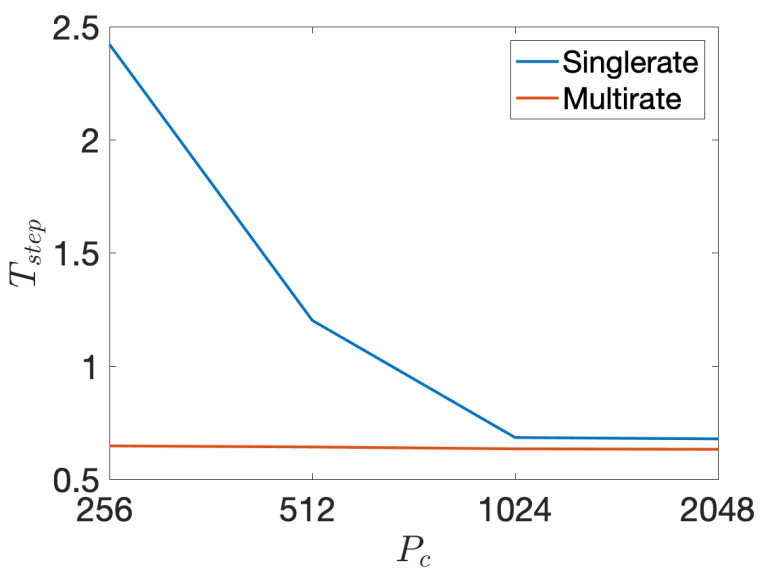

Figure 14: Variation of time to solution per timestep $\left(T_{\text {step }}\right)$ for the singlerate and multirate timestepping scheme with number of MPI ranks $\left(P_{c}\right)$ used for the subdomain $\left(\Omega^{c}\right)$ with slower time-scales. These tests were done using the buoyant plume problem (Section 4.2), where $E_{f}=55,480$ and $E_{c}=15,560$, and $P_{f}$ is fixed at 4096 MPI ranks.

data exchange at each (sub-)timestep.

Based on the results presented here, we conclude that load balance can be ensured for multirate timestepping-based calculations by choosing the MPI ranks for each subdomain such that

$$
\frac{P_{c}}{P_{f}} \approx \frac{E_{c}}{\eta E_{f}} .
$$

Though the above relationship might be constrained by the maximum memory available per MPI rank, as observed in the above test.

\section{Conclusion}

The current work discusses a novel parallel multirate timestepping scheme for the incompressible Navier-Stokes equations in nonconforming overlapping grids. This method scales to an arbitrary number of overlapping grids and is agnostic of the spatial discretization. The MTS method uses a timestep size based on the local CFL of each subdomain, which unlike the STS-based implementation, avoids unnecessary computation for the subdomain with slower time-scales $\left(\Omega^{c}\right)$. The MTS-based framework also requires fewer computational resources for $\Omega^{c}$ in comparison to STS-based framework because the INSE has to be integrated for fewer timesteps in $\Omega^{c}$. Using a problem with a known exact solution, we have demonstrated that the MTS method maintains the temporal convergence of the underlying timestepper. We have also demonstrated that the MTS method can accurately model complex turbulent flow using the example of a thermally-buoyant plume. This problem also shows the computational savings associated with the MTS-based method in comparison to an STS-based approach. In future work, we will be extending this method such that the timestep size and timestep ratios can dynamically change during the calculations based on the CFL of each subdomain, and accordingly load balance the calculation using (54) to further increase the computational savings associated with MTS. 


\section{Acknowledgments}

This work was supported by the U.S. Department of Energy, Office of Science, the Office of Advanced Scientific Computing Research, under Contract DE-AC02-06CH11357. This research used resources of the Argonne Leadership Computing Facility, which is a DOE Office of Science User Facility. An award of computer time on Blue Waters was provided by the National Center for Supercomputing Applications. Blue Waters is a sustained-petascale HPC and is a joint effort of the University of Illinois at Urbana-Champaign and its National Center for Supercomputing Applications. The Blue Waters sustained-petascale computing project is supported by the National Science Foundation (awards OCI-0725070 and ACI1238993) and the state of Illinois.

\section{References}

[1] K. Mittal, S. Dutta, P. Fischer, Nonconforming Schwarz-spectral element methods for incompressible flow, Computers \& Fluids (2019) 104237.

[2] P. M. Gresho, R. L. Lee, R. L. Sani, T. Stullich, Time-dependent FEM solution of the incompressible Navier-Stokes equations in two-and three-dimensions, Technical Report, California Univ., 1978.

[3] M. Rosenfeld, D. Kwak, M. Vinokur, A solution method for the unsteady incompressible navier-stokes equations in generalized coordinate systems, in: 26th Aerospace Sciences Meeting, 1988, p. 718.

[4] M. O. Deville, P. F. Fischer, E. H. Mund, High-order methods for incompressible fluid flow, volume 9, Cambridge University Press, 2002.

[5] A. Tomboulides, J. Lee, S. Orszag, Numerical simulation of low Mach number reactive flows, Journal of Scientific Computing 12 (1997) 139-167.

[6] H. Lewy, K. Friedrichs, R. Courant, Über die partiellen differenzengleichungen der mathematischen physik, Mathematische annalen 100 (1928) 32-74.

[7] A. Fabregat Tomàs, A. C. Poje, T. M. Özgökmen, W. K. Dewar, Dynamics of multiphase turbulent plumes with hybrid buoyancy sources in stratified environments, Physics of Fluids 28 (2016).

[8] W. D. Henshaw, Cgins user guide: An Overture solver for the incompressible NavierStokes equations on composite overlapping grids, Lawrence Livermore National Laboratory, Livermore, CA, USA, Software Manual LLNL-SM-455851 (2012).

[9] J. B. Angel, J. W. Banks, W. D. Henshaw, A high-order accurate FDTD scheme for Maxwell's equations on overset grids, in: Applied Computational Electromagnetics Society Symposium (ACES), 2018 International, IEEE, 2018, pp. 1-2.

[10] J. R. Rice, Split runge-kutta methods for simultaneous equations, Journal of Research of the National Institute of Standards and Technology 60 (1960). 
[11] C. Gear, Multirate methods for ordinary differential equations, Technical Report COO2383-0014, Illinois Univ., Urbana (USA). Dept. of Computer Science, 1974.

[12] C. W. Gear, D. Wells, Multirate linear multistep methods, BIT Numerical Mathematics 24 (1984) 484-502.

[13] J. F. Andrus, Numerical solution of systems of ordinary differential equations separated into subsystems, SIAM Journal on Numerical Analysis 16 (1979) 605-611.

[14] J. Andrus, Stability of a multi-rate method for numerical integration of ode's, Computers \& Mathematics with applications 25 (1993) 3-14.

[15] M. Günther, A. Kvaern $\varnothing$, P. Rentrop, Multirate partitioned runge-kutta methods, BIT Numerical Mathematics 41 (2001) 504-514.

[16] A. Verhoeven, E. J. W. Ter Maten, R. M. Mattheij, B. Tasić, Stability analysis of the BDF slowest-first multirate methods, International Journal of Computer Mathematics 84 (2007) 895-923.

[17] N. Godel, S. Schomann, T. Warburton, M. Clemens, Gpu accelerated adams-bashforth multirate discontinuous galerkin fem simulation of high-frequency electromagnetic fields, IEEE Transactions on magnetics 46 (2010) 2735-2738.

[18] C. Engstler, C. Lubich, Multirate extrapolation methods for differential equations with different time scales, Computing 58 (1997) 173-185.

[19] C. Dawson, R. Kirby, High resolution schemes for conservation laws with locally varying time steps, SIAM Journal on Scientific Computing 22 (2001) 2256-2281.

[20] E. M. Constantinescu, A. Sandu, Multirate timestepping methods for hyperbolic conservation laws, Journal of Scientific Computing 33 (2007) 239-278.

[21] B. Seny, J. Lambrechts, R. Comblen, V. Legat, J.-F. Remacle, Multirate time stepping for accelerating explicit discontinuous Galerkin computations with application to geophysical flows, International Journal for Numerical Methods in Fluids 71 (2013) 41-64.

[22] V. Savcenco, W. Hundsdorfer, J. Verwer, A multirate time stepping strategy for stiff ordinary differential equations, BIT Numerical Mathematics 47 (2007) 137-155.

[23] I. Rybak, J. Magiera, R. Helmig, C. Rohde, Multirate time integration for coupled saturated/unsaturated porous medium and free flow systems, Computational Geosciences 19 (2015) 299-309.

[24] M. Emmett, W. Zhang, J. B. Bell, High-order algorithms for compressible reacting flow with complex chemistry, Combustion Theory and Modelling 18 (2014) 361-387.

[25] C. J. Trahan, C. Dawson, Local time-stepping in Runge-Kutta discontinuous Galerkin finite element methods applied to the shallow-water equations, Computer Methods in Applied Mechanics and Engineering 217 (2012) 139-152. 
[26] S. Gupta, B. Wohlmuth, R. Helmig, Multi-rate time stepping schemes for hydrogeomechanical model for subsurface methane hydrate reservoirs, Advances in water resources 91 (2016) 78-87.

[27] C. Mikida, A. Klöckner, D. Bodony, Multi-rate time integration on overset meshes, arXiv preprint arXiv:1805.06607 (2018).

[28] A. Klöckner, High-performance high-order simulation of wave and plasma phenomena, Ph.D. thesis, Brown University, 2010.

[29] A. T. Patera, A spectral element method for fluid dynamics: laminar flow in a channel expansion, Journal of computational Physics 54 (1984) 468-488.

[30] P. Fischer, M. Schmitt, A. Tomboulides, Recent developments in spectral element simulations of moving-domain problems, in: Recent Progress and Modern Challenges in Applied Mathematics, Modeling and Computational Science, Springer, 2017, pp. 213-244.

[31] J. W. Lottes, P. F. Fischer, Hybrid multigrid/Schwarz algorithms for the spectral element method, Journal of Scientific Computing 24 (2005) 45-78.

[32] K. Mittal, Highly Scalable Solution of Incompressible Navier-Stokes equations using the Spectral Element Method with Overlapping Grids, Ph.D. thesis, University of Illinois at Urbana-Champaign, 2019.

[33] H. A. Schwarz, Ueber einen Grenzübergang durch alternirendes Verfahren, Zürcher u. Furrer, 1870.

[34] B. Smith, P. Bjorstad, W. Gropp, Domain decomposition: parallel multilevel methods for elliptic partial differential equations, Cambridge university press, 2004.

[35] B. Fornberg, A practical guide to pseudospectral methods, volume 1, Cambridge university press, 1998.

[36] Y. T. Peet, P. F. Fischer, Stability analysis of interface temporal discretization in grid overlapping methods, SIAM Journal on Numerical Analysis 50 (2012) 3375-3401.

[37] B. E. Merrill, Y. T. Peet, Effect of impinging wake turbulence on the dynamic stall of a pitching airfoil, AIAA Journal (2017) 4094-4112.

[38] B. E. Merrill, Y. T. Peet, Moving overlapping grid methodology of spectral accuracy for incompressible flow solutions around rigid bodies in motion, Journal of Computational Physics 390 (2019) 121-151.

[39] K. Mittal, S. Dutta, P. Fischer, Direct numerical simulation of rotating ellipsoidal particles using moving nonconforming schwarz-spectral element method, arXiv preprint arXiv:1912.04393 (2019).

[40] P. Fishcer, gslib/gslib, 2017. URL: https://github.com/gslib/gslib. 
[41] A. Noorani, G. Sardina, L. Brandt, P. Schlatter, Particle transport in turbulent curved pipe flow, Journal of Fluid Mechanics 793 (2016) 248-279.

[42] H. M. Tufo, P. F. Fischer, Fast parallel direct solvers for coarse grid problems, Journal of Parallel and Distributed Computing 61 (2001) 151-177.

[43] S. Dutta, K. Mittal, J. W. Lottes, P. Fischer, Efficient lagrangian particle tracking for high-order cfd in complex geometries, Book of Abstracts, NAHOMCon 2019 (2019) $37-38$.

[44] K. Mittal, S. Dutta, P. Fischer, Stability analysis of a singlerate and multirate predictorcorrector scheme for overlapping grids, arXiv preprint arXiv:2010.00118 (2020).

[45] H. J. Stetter, Improved absolute stability of predictor-corrector schemes, Computing 3 (1968) 286-296.

[46] E. Love, W. J. Rider, G. Scovazzi, Stability analysis of a predictor/multi-corrector method for staggered-grid lagrangian shock hydrodynamics, Journal of Computational Physics 228 (2009) 7543-7564.

[47] O. Walsh, Eddy solutions of the Navier-Stokes equations, in: The Navier-Stokes Equations II-Theory and Numerical Methods, Springer, 1992, pp. 306-309.

[48] C. M. Reddy, J. S. Arey, J. S. Seewald, S. P. Sylva, K. L. Lemkau, R. K. Nelson, C. A. Carmichael, C. P. McIntyre, J. Fenwick, G. T. Ventura, et al., Composition and fate of gas and oil released to the water column during the deepwater horizon oil spill, Proceedings of the National Academy of Sciences 109 (2012) 20229-20234.

[49] E. Kaminski, S. Tait, G. Carazzo, Turbulent entrainment in jets with arbitrary buoyancy, Journal of Fluid Mechanics 526 (2005) 361-376.

[50] M. Walter, C. Mertens, U. Stöber, C. R. German, D. R. Yoerger, J. Sültenfuß, M. Rhein, B. Melchert, E. T. Baker, Rapid dispersal of a hydrothermal plume by turbulent mixing, Deep Sea Research Part I: Oceanographic Research Papers 57 (2010) 931-945.

[51] W. Schmidt, Turbulent propagation of a stream of heated air, Z. Angew. Math. Mech 21 (1941) 265-278.

[52] J. Lavelle, Buoyancy-driven plumes in rotating, stratified cross flows: Plume dependence on rotation, turbulent mixing, and cross-flow strength, Journal of Geophysical Research: Oceans 102 (1997) 3405-3420.

[53] A. F. Tomàs, A. C. Poje, T. M. Özgökmen, W. K. Dewar, Effects of rotation on turbulent buoyant plumes in stratified environments, Journal of Geophysical Research: Oceans 121 (2016) 5397-5417.

[54] A. F. Tomàs, A. C. Poje, T. M. Özgökmen, W. K. Dewar, Numerical simulations of rotating bubble plumes in stratified environments, Journal of Geophysical Research: Oceans 122 (2017) 6795-6813. 
[55] P. K. Kundu, I. M. Cohen, Fluid mechanics, Elsevier, 2001.

[56] B. Morton, G. I. Taylor, J. S. Turner, Turbulent gravitational convection from maintained and instantaneous sources, Proceedings of the Royal Society of London. Series A. Mathematical and Physical Sciences 234 (1956) 1-23. 